\title{
EXISTENCE FOR A DEGENERATE CAUCHY PROBLEM
}

\author{
TUOMO KUUSI AND MIKKO PARVIAINEN
}

\begin{abstract}
We prove the existence of a solution to the degenerate parabolic Cauchy problem with a possibly unbounded Radon measure as an initial data. To accomplish this, we establish a priori estimates and derive a compactness result. We also show that the result is optimal in the Euclidian setting.
\end{abstract}

\section{INTRODUCTION}

We study the existence of solutions to the Cauchy problem

$$
\operatorname{div} \mathcal{A}(x, t, \nabla u)=\frac{\partial u}{\partial t}
$$

on $M \times(0, T)$ with a Radon measure $\nu$ as an initial trace. Here $M$ is a suitable Riemannian manifold satisfying doubling condition and the Poincaré inequality with uniform constants. As far as we know, our results are new even in $\mathbb{R}^{n} \times(0, T)$. We show that if the quantity

$$
|\|\nu\||=\limsup _{R \rightarrow \infty} R^{-p /(p-2)} \frac{|\nu|(B(x, R))}{\mu(B(x, R))}, \quad x \in M, \quad p>2,
$$

is bounded, then there exists a solution with the initial trace $\nu$ up to the time $T=C /|\|\nu\||^{2-p}$. Here $\mu$ is a doubling measure on $M$ and $|\nu|$ denotes the total variation of $\nu$. In particular, if $|\|\nu\||=0$, then the solution exists for all times. Furthermore, the existence result is sharp: We show that the existence fails for more general Radon measures in the Euclidean setting.

In [20], DiBenedetto and Herrero proved the existence and uniqueness for the evolutionary $p$-Laplace equation

$$
\frac{\partial u}{\partial t}=\operatorname{div}\left(|\nabla u|^{p-2} \nabla u\right), \quad p>2
$$

in the Euclidean setting. The existence of this important special case is included in our results. See also DiBenedetto [17]. For further related results we refer to Andreu et. el. [3], Blanchard-Murat [9], Boccardo et. al. [10], Rakotoson [33], and Manfredi-Vespri [31].

Our proof relies on a priori estimates and a compactness result. Starting from the weak formulation, we show that a bounded sequence of solutions has a subsequence that converges to a weak solution. It is worth noting that

Date: 31.10 .2008 .

2000 Mathematics Subject Classification. 35K55, 35K15, 35K65.

Key words and phrases. general nonlinear parabolic partial differential equation, initial value problem, measure initial data, optimal condition for existence, Riemannian manifold.

Department of Mathematics, Helsinki University of Technology, P.O.Box 1100, FIN02015 TKK (tuomo.kuusi@tkk.fi, mikko.parviainen@tkk.fi). 
the proof avoids Hölder estimates and the Arzelà-Ascoli theorem. By approximating the initial trace with compactly supported functions, we obtain approximative solutions which converge to a weak solution by the compactness result and a priori estimates. Due to a gradient estimate, this solution has the right initial trace. The optimality is a consequence of the global Harnack estimate.

We consider three types of a priori estimates providing boundedness for essential supremum of a subsolution, finite speed of propagation, and a bound for the gradient. In view of the Barenblatt solution [7], our estimates are optimal. The proofs for the a priori estimates apply modified Moser's iteration method and avoid the use of the scaling argument (cf. Choe-Lee [14]). In particular, the proofs have a local nature. For a priori estimates on the Riemannian manifolds, see also Dekkers [16] and Bonforte-Grillo [12]. Furthermore, the connection between parabolic equations and geometric properties of the manifolds, has been studied, for example, by Grigori'yan [23], Saloff-Coste [34], and Bakry et. al. [6].

Classical results in, for example, Widder [38], state that if initial data satisfies the exponential growth condition, then there exists a solution to the heat equation. In [4], Aronson proved the optimality of such a condition for equations with bounded measurable coefficients. For the porous medium equation the existence of a solution was established by Benilan, Crandall and Pierre in [8] provided that the initial trace satisfies a suitable growth condition. Later, Aronson and Caffarelli obtained the necessity of such a condition in [5]. The uniqueness of the solution is due to Dahlberg and Kenig [15].

\section{Preliminaries}

Let $M$ be a smooth, noncompact, connected, and complete Riemannian manifold without a boundary and of dimension $n$. We use path metric throughout the work and assume that there exists a doubling measure $\mu$, i.e. we have a constant $C_{\mu} \geq 1$ such that

$$
\mu(B(z, 2 R)) \leq C_{\mu} \mu(B(z, R))
$$

for every ball $B(z, 2 R)$ in $M$. The dimension of the measure is $d_{\mu}=\log _{2} C_{\mu}$. Let $T_{x} M$ be the tangent space to $M$ at the point $x$. Because $M$ is a Riemannian manifold, we are given on each $T_{x} M$ a scalar product $\langle\cdot, \cdot\rangle_{x}$. We usually surpress the dependence on $x$. The space $T M$ is defined in a usual way as the disjoint union of the tangent spaces $T_{x} M$.

Let $\Omega$ be an open bounded (in the sense of the path metric) set in $M$. We define the Sobolev norm

$$
\|f\|_{1, p, \Omega}=\left(\int_{\Omega}\left(|f|^{p}+|\nabla f|^{p}\right) d \mu\right)^{1 / p}
$$

where $f \in C^{\infty}(M)$ and $|\nabla f|=\langle\nabla f, \nabla f\rangle^{1 / 2}$, see for example do Carmo [21] and Saloff-Coste [34] for more details.

The Sobolev space $W^{1, p}(\Omega)$ is defined to be the closure of $C^{\infty}(\Omega)$ with respect to the Sobolev norm. Similarly, the Sobolev space with zero boundary values, denoted by $W_{0}^{1, p}(\Omega)$, is the closure of $C_{0}^{\infty}(\Omega)$. A function belongs to the local Sobolev space $W_{l o c}^{1, p}(\Omega)$ if it belongs to $W^{1, p}\left(\Omega^{\prime}\right)$ for every open 
subset $\Omega^{\prime}$ whose closure is a compact subset of $\Omega$. For further discussion on the Sobolev spaces on Riemannian manifolds, see, for example, Hebey [24].

Furthermore, we assume that $M$ satisfies a $(1, p)$-Poincaré inequality

$$
f_{B(z, R)}\left|f-f_{B(z, R)}\right| d \mu \leq C_{p} f_{B(z, R)}|\nabla f|^{p} d \mu
$$

with a uniform constant $C_{p}>0$. Here the integral average of $f$ is denoted by

$$
f_{B(z, R)}=f_{B(z, R)} f d \mu=\frac{1}{\mu(B(z, R))} \int_{B(z, R)} f d \mu .
$$

The $(1, p)$-Poincaré inequality leads to the Sobolev inequality for the Sobolev functions with zero boundary values. Indeed, suppose that $u$ is in $W_{0}^{1, p}(B(z, R))$. Then there exists constants $C=C\left(p, C_{\mu}, C_{p}\right)$ and $\kappa=\kappa\left(p, C_{\mu}, C_{p}\right)>1$ such that

$$
\left(f_{B(z, R)}|v|^{\kappa p} d \mu\right)^{1 /(\kappa p)} \leq C R\left(f_{B(z, R)}|\nabla v|^{p} d \mu\right)^{1 / p} .
$$

Here $\kappa=d_{\mu} /\left(d_{\mu}-p\right)$, if $p<d_{\mu}$ and $\kappa \in(p, \infty)$, if $p \geq d_{\mu}$. Recall that $d_{\mu}$ is the dimension of the measure. For the proof we refer, for example, to Kinnunen-Shanmugalingam [27].

We denote by $L^{p}\left(t_{1}, t_{2} ; W^{1, p}(\Omega)\right), t_{1}<t_{2}$, the parabolic Sobolev space. This space consists of functions $u$ such that for almost every $t, t_{1}<t<t_{2}$, the function $x \mapsto u(x, t)$ belongs to $W^{1, p}(\Omega)$ and the norm

$$
\left(\int_{t_{1}}^{t_{2}} \int_{\Omega}|u(x, t)|^{p}+|\nabla u(x, t)|^{p} d \mu(x) d t\right)^{1 / p}
$$

is finite. The definitions for $L_{l o c}^{p}\left(t_{1}, t_{2} ; W_{l o c}^{1, p}(\Omega)\right)$ and $L^{p}\left(t_{1}, t_{2} ; W_{0}^{1, p}(\Omega)\right)$ are analogous. Finally, the space $C\left(\left(t_{1}, t_{2}\right) ; L^{q}(\Omega)\right), q=1,2$, comprises of all the functions $u$ such that for $t_{1}<s, t<t_{2}$, we have

$$
\int_{\Omega}|u(x, t)-u(x, s)|^{q} d \mu(x) \rightarrow 0
$$

as $s \rightarrow t$.

We study the Cauchy problem

$$
\left\{\begin{aligned}
\operatorname{div}(\mathcal{A}(x, t, \nabla u)) & =\frac{\partial u}{\partial t} \quad \text { in } \quad M \times(0, T), \\
u(\cdot, 0) & =\nu
\end{aligned}\right.
$$

where $\nu$ is a Radon measure (for a precise formulation, see Definition 2.5). The solution $u$ satisfies the initial condition in the sense of distributions, i.e.

$$
\lim _{t \rightarrow 0} \int_{M} u(x, t) \varphi(x) d \mu(x)=\int_{M} \varphi(x) d \nu(x)
$$

for all $\varphi \in C_{0}^{\infty}(M)$. We say that $\nu$ is the initial trace of $u$.

The function $\mathcal{A}$ (for which $\mathcal{A}(x, t, \xi) \in T_{x} M$, where $(x, t) \in M \times \mathbb{R}^{+}$, and $\left.\xi \in T_{x} M\right)$ satisfies Carathéodory, monotonicity, and $p$-growth conditions, i.e.,

(1) $(x, t) \mapsto \mathcal{A}(x, t, X)$ is measurable for all measurable vector fields $X$, 
(2) $\xi \mapsto \mathcal{A}(x, t, \xi), \xi \in T_{x} M$, is continuous for almost every $(x, t) \in$ $M \times \mathbb{R}^{+}$

(3) there exists $0<\alpha \leq \beta<\infty$ such that

$$
\begin{aligned}
& \langle\mathcal{A}(x, t, \xi), \xi\rangle \geq \alpha|\xi|^{p}, \\
& |\mathcal{A}(x, t, \xi)| \leq \beta|\xi|^{p-1},
\end{aligned}
$$

for almost every $(x, t) \in M \times \mathbb{R}^{+}$and for every $\xi \in T_{x} M$.

(4) $\mathcal{A}$ is strictly monotone, that is,

$$
\langle\mathcal{A}(x, t, \xi)-\mathcal{A}(x, t, \zeta), \xi-\zeta\rangle>0
$$

for almost every $(x, t)$ in $M \times \mathbb{R}^{+}$, and every $\xi, \zeta$ in $T_{x} M, \xi \neq \zeta$.

We denote

$$
M_{\tau_{1}, \tau_{2}}=M \times\left(\tau_{1}, \tau_{2}\right), \quad \Omega_{\tau_{1}, \tau_{2}}=\Omega \times\left(\tau_{1}, \tau_{2}\right) .
$$

The notation $U \Subset \Omega$ denotes that $U$ is a bounded subset of $\Omega$ and the closure of $U$ belongs to $\Omega$.

The definition of the weak solution is local. In particular, it does not impose any condition on the initial data.

Definition 2.5. Let $\Xi$ be an open set in $M \times \mathbb{R}$. A measurable function $u: \Xi \rightarrow \mathbb{R}$ is a weak subsolution (supersolution), if whenever $\Omega_{\tau_{1}, \tau_{2}} \Subset \Xi$, then

$$
u \in L^{p}\left(\tau_{1}, \tau_{2} ; W^{1, p}(\Omega)\right)
$$

and it satisfies the integral inequality

$$
\int_{\tau_{1}}^{\tau_{2}} \int_{\Omega}\langle\mathcal{A}(x, t, \nabla u), \nabla \phi\rangle d \mu d t-\int_{\tau_{1}}^{\tau_{2}} \int_{\Omega} u \frac{\partial \phi}{\partial t} d \mu d t \leq(\geq) 0
$$

for every non-negative test function $\phi \in C_{0}^{\infty}\left(\Omega_{\tau_{1}, \tau_{2}}\right)$. A weak solution is a weak sub- and supersolution and (2.6) holds with equality for all test functions $\phi \in C_{0}^{\infty}\left(\Omega_{\tau_{1}, \tau_{2}}\right)$.

Remark 2.7. By the growth bounds (2.3) and the approximation, the functional $f$ defined via

$$
\langle f, \phi\rangle=\int_{\tau_{1}}^{\tau_{2}} \int_{\Omega}\langle\mathcal{A}(x, t, \nabla u), \nabla \phi\rangle d \mu d t
$$

belongs to the dual space of $L^{p}\left(\tau_{1}, \tau_{2} ; W_{0}^{1, p}(\Omega)\right)$, whenever $\Omega_{\tau_{1}, \tau_{2}} \Subset \Xi$. Thus, if $u$ is a weak solution, then the equation implies that the time derivative of $u$ belongs to the same dual space. Consequently, $u$ belongs to $C\left(\left[\tau_{1}, \tau_{2}\right], L^{2}(\Omega)\right)$, see, for example, Lions [30] and also Showalter [35].

Later we shall consider initial and boundary value problem with $L^{2}$ - initial data in bounded $\Omega$. In that case, a weak solution belongs to $C\left([0, T), L^{2}(\Omega)\right)$, see (5.1) and (5.2). The $L^{2}$-continuity also extends to the Cauchy problem with compactly supported $L^{2}$ initial data as we shall show in Theorem 5.4. However, with a radon measure as an initial data, see Theorem 5.6, we can not assume the $L^{2}$-continuity at $t=0$.

The parabolic theory differs from the elliptic theory when proving estimates for the weak solutions. One often needs a test function depending 
on $u$ itself, but the time derivative of a test function may not exist as a function. We treat this difficulty by using the standard mollification

$$
\phi_{\delta}(x, t)=\int_{\mathbb{R}} \phi(x, s) \zeta_{\delta}(s-t) d s, \quad x \in M, \quad \tau_{1}<t<\tau_{2},
$$

where $\phi \in C^{\infty}\left(\Omega_{\tau_{1}, \tau_{2}}\right)$ and $\zeta_{\delta}(s)$ is the standard mollifier, whose support is contained in $(-\delta, \delta), \delta<\operatorname{dist}\left(\operatorname{spt}(\phi), \partial \Omega_{\tau_{1}, \tau_{2}}\right)$. Next we insert the test function into (2.6), change variables, apply Fubini's theorem, integrate by parts, and obtain the following regularized equation

$$
\int_{\tau_{1}}^{\tau_{2}} \int_{\Omega} \frac{\partial u_{\delta}}{\partial t} \phi d \mu d t+\int_{\tau_{1}}^{\tau_{2}} \int_{\Omega}\left\langle\mathcal{A}(x, t, \nabla u), \nabla \phi_{\delta}\right\rangle d \mu d t \leq(\geq) 0,
$$

where $u_{\delta}$ is the mollification in the time direction.

\section{A PRIORI EStimates}

This section deals with three type of a priori estimates providing boundedness for essential supremum of a subsolution, finite speed of propagation, and a bound for the gradient. Theorem 3.1, the main result of the section, is later used in proving the existence of a Cauchy problem. In particular, the estimates help us in obtaining preliminary existence and compactness results as well as the connection to the initial data. The proofs apply Moser's iteration method and have a local nature. A priori estimates have plenty of further applications: Together with the compactness results, they imply an existence of the universal bound for homogenous Dirichlet problems (cf. Vázquez [37] in a different context). A priori estimates also play an important role in further regularity results, see for example Acerbi-Mingione [1] and DiBenedetto [17].

In [17], a priori estimates are derived using De Giorgi's method in the Euclidian setting. Furthermore, Dekkers proves the finite speed of propagation on Riemannian manifolds applying De Giorgi's method in [16]. However, the propagation speed obtained there is not optimal as the Barenblatt solution shows. Bonforte and Grillo consider the porous medium equation on a compact Riemannian manifold in [12].

Finally, we note that the estimates hold whenever the Riemannian manifold supports the local doubling condition and the Poincaré inequality. In particular, when the Ricci curvature is bounded below by $-K g, K>0$, then the constants $C_{\mu}$ and $C_{p}$ behave like a power of $\exp (\sqrt{K} R)$, where $R$ is the radius of the ball, see Chavel [13]. Hence the theorem below applies as such whenever $R \leq 1 / \sqrt{K}$.

Theorem 3.1. Let $u$ be a non-negative weak subsolution in $B\left(x_{0}, 2 R\right) \times$ $\left(t_{0}, t_{0}+T\right)$ and denote

$$
N_{s}=\operatorname{essip}_{t_{0}<t<t_{0}+s} f_{B\left(x_{0}, 2 R\right)} u d \mu
$$

$0<s \leq T$. Then

i) The subsolution is bounded:

$$
\underset{Q}{\operatorname{ess} \sup } u \leq C\left(\frac{R^{p}}{\tau}\right)^{1 /(p-2)}+C \frac{\tau}{R^{p}} N_{\tau}^{p-1},
$$


where $Q=B\left(x_{0}, R\right) \times\left(t_{0}+\tau / 2, t_{0}+\tau\right)$ and $0<\tau \leq T$.

ii) Disturbances have a finite speed of propagation: Assume that $u$ has zero initial data in sense that

$$
\lim _{h \rightarrow 0} f_{t_{0}+h}^{t_{0}+2 h} \int_{B\left(x_{0}, 2 R\right)} u^{2} d \mu d t=0 .
$$

Then $u=0$ almost everywhere in $B\left(x_{0}, R\right) \times\left(t_{0}, t_{0}+\tau\right)$, where

$$
\tau=\min \left\{T, R^{p} N_{T}^{2-p} / C\right\} .
$$

iii) For the gradient, we have the estimate

$$
\begin{aligned}
& \quad \int_{t_{0}}^{t_{0}+\tau} f_{B\left(x_{0}, R\right)}|\nabla u|^{p-1} d \mu d t \leq C\left(\left(R^{d_{\mu}} N_{\tau}\right)^{p-2} \tau\right)^{1 / \lambda} N_{\tau} \\
& \lambda=d_{\mu}(p-2)+p \text {, which holds for every } 0<\tau<T \text { such that } \\
& \tau \leq R^{p} N_{\tau}^{2-p} .
\end{aligned}
$$

Above $C=C\left(p, \alpha, \beta, C_{\mu}, C_{p}\right)$ is a positive constant.

Remark 3.5. If $u$ is a solution in Theorem 3.1, then the results hold with $u$ replaced by $|u|$ and $N$ replaced by

$$
N=\max \left(N^{+}, N^{-}\right), \quad N^{ \pm}=\underset{t_{0}<t<t_{0}+T}{\operatorname{ess} \sup _{B\left(x_{0}, 2 R\right)}} \max ( \pm u, 0) d \mu,
$$

or alternatively

$$
N=\operatorname{essip}_{t_{0}<t<t_{0}+T} \int_{B\left(x_{0}, 2 R\right)}|u| d \mu .
$$

This is because $\max ( \pm u, 0)$ is a subsolution and $|u|=\max (u, 0)+$ $\max (-u, 0)$. Observe that the equation may change, but its structure remains the same.

The proof of the Theorem 3.1 is based on the Moser's iteration method in which we utilize the Sobolev inequality and the following Caccioppoli estimate with possibly large $\varepsilon$. However, a subsolution is known to be integrable only up to the power $p$. We avoid by difficulties using the truncation in the proof of the Caccioppoli inequality.

Lemma 3.6. Suppose that $u$ is a non-negative subsolution in $\Omega_{\tau_{1}, \tau_{2}}$. Then we have

$$
\begin{aligned}
\int_{\tau_{1}}^{\tau_{2}} \int_{\Omega}|\nabla u|^{p} u^{-1+\varepsilon} \phi^{p} d \mu d t+\frac{p}{\alpha \varepsilon(1+\varepsilon)} \operatorname{ess~sup}_{\tau_{1}<t<\tau_{2}} \int_{\Omega} u^{1+\varepsilon} \phi^{p} d \mu \\
\leq\left(\frac{\beta p}{\alpha}\right)^{p} \int_{\tau_{1}}^{\tau_{2}} \int_{\Omega} u^{p-1+\varepsilon}|\nabla \phi|^{p} d \mu d t \\
\quad+\frac{p}{\alpha(1+\varepsilon)} \int_{\tau_{1}}^{\tau_{2}} \int_{\Omega} u^{1+\varepsilon}\left(\frac{\partial \phi^{p}}{\partial t}\right)_{+} d \mu d t
\end{aligned}
$$

for every non-negative $\phi \in C_{0}^{\infty}\left(\Omega_{\tau_{1}, \tau_{2}}\right)$ and $\varepsilon \geq 1$.

Proof. To begin with, we define $\chi_{\tau_{1}, \tau}^{h}(t)$, where $\tau_{1}<\tau<\tau_{2}$, to be a piecewise linear approximation of a characteristic function such that

$$
\begin{gathered}
\chi_{\tau_{1}, \tau}^{h}(t)=1 \quad \text { as } \quad \tau_{1}+2 h \leq t \leq \tau-2 h, \\
\chi_{\tau_{1}, \tau}^{h}(t)=0 \quad \text { as } \quad t \leq \tau_{1}+h \quad \text { or } \quad t \geq \tau-h,
\end{gathered}
$$


and

$$
\left|\left(\chi_{\tau_{1}, \tau}^{h}(t)\right)^{\prime}\right| \leq \frac{1}{h}
$$

Let $\delta<h / 4$ and choose $\phi \in C_{0}^{\infty}\left(\Omega_{\tau_{1}, \tau_{2}}\right)$. We insert the test function

$$
\eta=\min \left\{u_{\delta}^{-1+\varepsilon}, k^{-1+\varepsilon}\right\} u_{\delta} \phi^{p} \chi_{\tau_{1}, \tau}^{h} .
$$

into (2.8), where the subscript $\delta$ refers to the mollification. The test function $\eta$ is admissible due to the approximation. We obtain

$$
\int_{\tau_{1}}^{\tau_{2}} \int_{\Omega} \frac{\partial u_{\delta}}{\partial t} \eta d \mu d t+\int_{\tau_{1}}^{\tau_{2}} \int_{\Omega}\left\langle\mathcal{A}(x, t, \nabla u), \nabla \eta_{\delta}\right\rangle d \mu d t \leq 0 .
$$

It follows by the properties of the standard mollifiers that

$$
\left\langle\mathcal{A}(x, t, \nabla u), \nabla \eta_{\delta}\right\rangle \rightarrow\left\langle\mathcal{A}(x, t, \nabla u), \nabla\left(\min \left\{u^{-1+\varepsilon}, k^{-1+\varepsilon}\right\} u \phi^{p} \chi_{\tau_{1}, \tau}^{h}\right)\right\rangle
$$

in $L^{1}\left(\Omega_{t_{1}, t_{2}}\right)$ as $\delta \rightarrow 0$. When $u<k$, growth conditions (2.3) imply

$$
u^{\varepsilon-1}\langle\mathcal{A}(x, t, \nabla u), \nabla u\rangle \geq \alpha|\nabla u|^{p} u^{\varepsilon-1}
$$

and

$$
\begin{aligned}
& \frac{p}{\varepsilon} u^{\varepsilon} \phi^{p-1}\langle\mathcal{A}(x, t, \nabla u), \nabla \phi\rangle \geq-\frac{\beta p}{\varepsilon}|\nabla u|^{p-1} \phi^{p-1}|\nabla \phi| u^{\varepsilon} \\
& =-\alpha\left(|\nabla u| u^{(-1+\varepsilon) / p} \phi\right)^{p-1}\left(\frac{\beta p}{\alpha \varepsilon} u^{(p-1+\varepsilon) / p}|\nabla \phi|\right) \\
& \geq-\frac{(p-1)}{p} \alpha|\nabla u|^{p} u^{-1+\varepsilon} \phi^{p}-\frac{\alpha}{p}\left(\frac{\beta p}{\alpha \varepsilon}\right)^{p} u^{p-1+\varepsilon}|\nabla \phi|^{p} .
\end{aligned}
$$

Here we have also applied Young's inequality. Thus we arrive at

$$
\begin{aligned}
& \frac{p}{\alpha \varepsilon}\langle\mathcal{A}(x, t, \nabla u)\left., \nabla\left(\min \left\{u^{-1+\varepsilon}, k^{-1+\varepsilon}\right\} u \phi^{p} \chi_{\tau_{1}, \tau}^{h}\right)\right\rangle \\
& \geq|\nabla u|^{p} u^{-1+\varepsilon} \phi^{p} \chi_{\tau_{1}, \tau}^{h}-\left(\frac{\beta p}{\alpha \varepsilon}\right)^{p} u^{p-1+\varepsilon}|\nabla \phi|^{p} \chi_{\tau_{1}, \tau}^{h} .
\end{aligned}
$$

Similarly, when $u \geq k$, we have

$$
\frac{p}{\alpha}\langle\mathcal{A}(x, t, \nabla u), \nabla \eta\rangle \geq|\nabla u|^{p} k^{-1+\varepsilon} \phi^{p} \chi_{\tau_{1}, \tau}^{h}-\left(\frac{\beta p}{\alpha}\right)^{p} u^{p} k^{-1+\varepsilon}|\nabla \phi|^{p} \chi_{\tau_{1}, \tau}^{h} .
$$

Next we define an auxiliary function

$$
\begin{aligned}
g_{k}(s) & =\int_{0}^{s} \min \left\{r^{-1+\varepsilon}, k^{-1+\varepsilon}\right\} r d r \\
& =\frac{1}{1+\varepsilon} s^{1+\varepsilon} \chi_{(0, k]}(s)+k^{-1+\varepsilon}\left(\frac{k^{2}}{1+\varepsilon}+\frac{s^{2}-k^{2}}{2}\right) \chi_{(k,+\infty)}(s)
\end{aligned}
$$

to simplify the calculations. In particular, observe that

$$
\frac{\partial u_{\delta}}{\partial t} \min \left\{u_{\delta}^{-1+\varepsilon}, k^{-1+\varepsilon}\right\} u_{\delta}=\frac{\partial g_{k}\left(u_{\delta}\right)}{\partial t} .
$$

Hence the integration by parts yields

$$
\int_{\tau_{1}}^{\tau_{2}} \int_{\Omega} \frac{\partial u_{\delta}}{\partial t} \eta d \mu d t=-\int_{\tau_{1}}^{\tau_{2}} \int_{\Omega} g_{k}\left(u_{\delta}\right) \frac{\partial\left(\phi^{p} \chi_{\tau_{1}, \tau}^{h}\right)}{\partial t} d \mu d t
$$


Thus

$$
\begin{aligned}
& \int_{\tau_{1}}^{\tau_{2}} \int_{\Omega} \frac{\partial u_{\delta}}{\partial t} \eta d \mu d t \\
& \quad \rightarrow-\int_{\tau_{1}}^{\tau_{2}} \int_{\Omega} g_{k}(u) \chi_{\tau_{1}, \tau}^{h} \frac{\partial \phi^{p}}{\partial t} d \mu d t-\int_{\tau_{1}}^{\tau_{2}} \int_{\Omega} g_{k}(u) \phi^{p} \frac{\partial \chi_{\tau_{1}, \tau}^{h}}{\partial t} d \mu d t
\end{aligned}
$$

as $\delta \rightarrow 0$. Furthermore, we have

$$
\begin{aligned}
\int_{\tau_{1}}^{\tau_{2}} \int_{\Omega} g_{k}(u) \phi^{p} \frac{\partial \chi_{\tau_{1}, \tau}^{h}}{\partial t} d \mu d t & \\
& =-f_{\tau-2 h}^{\tau-h} \int_{\Omega} g_{k}(u) \phi^{p} d \mu d t+f_{\tau_{1}+h}^{\tau_{1}+2 h} \int_{\Omega} g_{k}(u) \phi^{p} d \mu d t
\end{aligned}
$$

Almost every $\tau$ is a Lebesgue instant, that is,

$$
\lim _{h \rightarrow 0} f_{\tau-2 h}^{\tau-h} \int_{K}(u(x, t)-u(x, \tau))^{2} d \mu d t=0
$$

for all compact sets $K$ in $\Omega$. This and the dominated convergence theorem imply that

$$
\int_{\tau_{1}}^{\tau_{2}} \int_{\Omega} g_{k}(u) \phi^{p} \frac{\partial \chi_{\tau_{1}, \tau}^{h}}{\partial t} d \mu d t \rightarrow-\int_{\Omega} g_{k}(u(x, \tau)) \phi^{p}(x, \tau) d \mu(x)
$$

as $h \rightarrow 0$ for almost every $k$. Observe that the initial term disappears due to the compact support of $\phi$.

We collect the terms and divide the result by $\alpha \varepsilon / p$. Note that $g_{k}(s)$ is monotone increasing with respect to $k$. Thus, as $k \rightarrow \infty$, by the monotone convergence theorem it follows that

$$
\begin{aligned}
0 \geq & \int_{\tau_{1}}^{\tau_{2}} \int_{\Omega}|\nabla u|^{p} u^{-1+\varepsilon} \phi^{p} d \mu d t-\left(\frac{\beta p}{\alpha}\right)^{p} \int_{\tau_{1}}^{\tau_{2}} \int_{\Omega} u^{p-1+\varepsilon}|\nabla \phi|^{p} d \mu d t \\
& -\frac{p}{\alpha(1+\varepsilon)} \int_{\tau_{1}}^{\tau_{2}} \int_{\Omega} u^{1+\varepsilon}\left(\frac{\partial \phi^{p}}{\partial t}\right)_{+} d \mu d t \\
& +\frac{p}{\alpha \varepsilon(1+\varepsilon)} \int_{\Omega} u^{1+\varepsilon}(x, \tau) \phi^{p}(x, \tau) d \mu(x)
\end{aligned}
$$

for all Lebesgue instants $\tau_{1}<\tau<\tau_{2}$. To conclude the proof, let $\rho>0$. We may choose $\tau_{1}<\tau<\tau_{2}$ such that

$$
\int_{\Omega} u^{1+\varepsilon}(x, \tau) \phi^{p}(x, \tau) d \mu(x) \geq \operatorname{ess~sup}_{\tau_{1}<t<\tau_{2}} \int_{\Omega} u^{1+\varepsilon} \varphi^{p} d \mu-\rho .
$$

This finishes the proof, since $\rho$ is arbitrary.

Remark 3.9. A slight modification of the proof gives the result also for $0<\varepsilon<1$ with a different constants. Indeed, we may first add a positive constant $\sigma$ to $u$. This guarantees that the test function $\eta=u_{\delta}^{\varepsilon} \phi^{p} \chi_{\tau_{1}, \tau}^{h}$ is admissible due to the approximation. In particular, we may omit the 
truncation. Following the proof, we end up with

$$
\begin{aligned}
0 \geq & \int_{\tau_{1}}^{\tau_{2}} \int_{\Omega}|\nabla u|^{p} u^{-1+\varepsilon} \phi^{p} d \mu d t-\left(\frac{\beta p}{\varepsilon \alpha}\right)^{p} \int_{\tau_{1}}^{\tau_{2}} \int_{\Omega} u^{p-1+\varepsilon}|\nabla \phi|^{p} d \mu d t \\
& -\frac{p}{\alpha \varepsilon(1+\varepsilon)} \int_{\tau_{1}}^{\tau_{2}} \int_{\Omega} u^{1+\varepsilon}\left(\frac{\partial \phi^{p}}{\partial t}\right)_{+} d \mu d t \\
& +\frac{p}{\alpha \varepsilon(1+\varepsilon)} \int_{\Omega} u^{1+\varepsilon}(x, \tau) \phi^{p}(x, \tau) d \mu(x) .
\end{aligned}
$$

The result then follows by the monotone convergence theorem as $\sigma \rightarrow 0$.

Remark 3.10. Suppose that $u$ has zero initial data, i.e. $\tau_{1}$ is a Lebesgue instant and

$$
\lim _{h \rightarrow 0} f_{\tau_{1}+h}^{\tau_{1}+2 h} \int_{\Omega} u^{2}(x, t) d \mu d t=0 .
$$

We follow the previous proof and choose the test function $\phi$ to depend only on $x$. Consequently, we obtain the estimate

$$
\begin{aligned}
& \int_{\tau_{1}}^{\tau_{2}} \int_{\Omega}|\nabla u|^{p} u^{-1+\varepsilon} \psi^{p} d \mu d t+\frac{p}{\alpha \varepsilon(1+\varepsilon)} \operatorname{ess~sup}_{\tau_{1}<t<\tau_{2}} \int_{\Omega} u^{1+\varepsilon} \psi^{p} d \mu \\
& \quad \leq\left(\frac{\beta p}{\alpha}\right)^{p} \int_{\tau_{1}}^{\tau_{2}} \int_{\Omega} u^{p-1+\varepsilon}|\nabla \psi|^{p} d \mu d t
\end{aligned}
$$

for all non-negative $\psi \in C_{0}^{\infty}(\Omega)$.

Remark 3.11. Let $u$ be a non-negative subsolution in $\Omega_{\tau_{1}, \tau_{2}}$. Suppose that it has zero boundary values in Sobolev sense, i.e.

$$
u \in L^{p}\left(t_{1}, t_{2} ; W_{0}^{1, p}(\Omega)\right)
$$

for all $\tau_{1}<t_{1}<t_{2}<\tau_{2}$. We choose $\phi$ to depend only on $t$. Then $\eta$ defined in (3.7) is an admissible test funtion for all $\varepsilon \geq 1$. Thus we obtain

$$
\begin{gathered}
\int_{\tau_{1}}^{\tau_{2}} \int_{\Omega}|\nabla u|^{p} u^{-1+\varepsilon} \phi^{p} d \mu d t+\frac{1}{\alpha \varepsilon(1+\varepsilon)} \operatorname{ess~sup}_{\tau_{1}<t<\tau_{2}} \int_{\Omega} u^{1+\varepsilon} \phi^{p} d \mu \\
\leq \frac{1}{\alpha(1+\varepsilon)} \int_{\tau_{1}}^{\tau_{2}} \int_{\Omega} u^{1+\varepsilon}\left(\frac{\partial \phi^{p}}{\partial t}\right)_{+} d \mu d t
\end{gathered}
$$

for all $\varepsilon \geq 1$. In particular, by choosing $\varepsilon=1$, we get

$$
2 \alpha \int_{\tau}^{\tau_{2}} \int_{\Omega}|\nabla u|^{p} \phi^{p} d \mu d t+\operatorname{essup}_{\tau<t<\tau_{2}} \int_{\Omega} u^{2} d \mu \leq \int_{\Omega} u^{2}(x, \tau) d \mu(x)
$$

for almost every $\tau_{1}<\tau<\tau_{2}$.

Remark 3.13. If $u$ is a non-negative subsolution in an open set containing compactly $\Omega_{t_{1}, t_{2}}$, where $t_{1}$ and $t_{2}$ are Lebesgue instants. We may then proceed as in the proof of Lemma 3.6 and obtain

$$
\begin{aligned}
\int_{\Omega} u\left(x, t_{2}\right) \phi(x) d \mu(x) \leq & \int_{\Omega} u\left(x, t_{1}\right) \phi(x) d \mu(x) \\
& -\int_{t_{1}}^{t_{2}} \int_{\Omega}\langle\mathcal{A}(x, t, \nabla u), \nabla \phi\rangle d \mu d t
\end{aligned}
$$

where $\phi \in C_{0}^{\infty}(\Omega)$. 
We continue the proof of Theorem 3.1 by showing the the boundedness of the weak subsolutions via Moser's iteration method. Observe that we avoid the use of the scaling argument. Therefore, we pay extra attention on the bookkeeping of the geometric ratio $R^{p} / T$.

Lemma 3.15. Let $u$ be a subsolution in $B\left(x_{0}, R\right) \times\left(t_{0}-T, t_{0}\right)$. Suppose that

$$
u^{p-2}(x, t) \geq \frac{R^{p}}{T} \quad \text { for all } \quad(x, t) \in B\left(x_{0}, R\right) \times\left(t_{0}-T, t_{0}\right) .
$$

Let $\delta_{0}>0$. Then there exist constants $C_{i}=C_{i}\left(p, \alpha, \beta, C_{\mu}, C_{p}, \delta_{0}\right), i=1,2$, such that

$$
\operatorname{esssup}_{B\left(x_{0}, \sigma R\right) \times\left(t_{0}-\sigma^{p} T, t_{0}\right)} u \leq\left(\frac{T}{R^{p}} \frac{C_{1}}{(1-\sigma)^{C_{2}}} f_{t_{0}-T}^{t_{0}} f_{B\left(x_{0}, R\right)} u^{p-2+\delta} d \mu d t\right)^{1 / \delta}
$$

for every $\delta \geq \delta_{0}$ and $0<\sigma<1$.

Proof. We set

$$
R_{j}=\sigma R+(\rho-\sigma) R 2^{-j}, \quad T_{j}=T \frac{R_{j}^{p}}{R^{p}}=\left(\sigma+(\rho-\sigma) 2^{-j}\right)^{p} T,
$$

$0<\sigma<\rho<1$, and

$$
B_{j}=B\left(x_{0}, R_{j}\right), \quad \Gamma_{j, h}=\Gamma_{j}=\left(t_{0}-T_{j}, t_{0}-h\left(T_{0}-T_{j}\right)\right), \quad Q_{j}=B_{j} \times \Gamma_{j},
$$

where $0<h<\sigma^{p} / 4$. We choose test functions $\phi_{j} \in C_{0}^{\infty}\left(Q_{j}\right)$ such that $0 \leq \phi_{j} \leq 1, \phi_{j}=1$ in $Q_{j+1}$, and

$$
\left|\nabla \phi_{j}\right| \leq \frac{C 2^{j}}{R(\rho-\sigma)}, \quad\left(\frac{\partial \phi_{j}^{p}}{\partial t}\right)_{+} \leq \frac{C 2^{j p}}{T(\rho-\sigma)^{p}}
$$

Let $\varepsilon \geq 1$. An application of Hölder's inequality yields

$$
\begin{aligned}
\int_{\Gamma_{j+1}} & f_{B_{j+1}} u^{(1+\varepsilon)(\kappa-1) / \kappa+p-1+\varepsilon} d \mu d t \\
\leq & \int_{\Gamma_{j+1}}\left(f_{B_{j+1}} u^{1+\varepsilon} \phi_{j}^{p} d \mu\right)^{(\kappa-1) / \kappa}\left(f_{B_{j+1}}\left(u^{(p-1+\varepsilon) / p} \phi_{j}\right)^{\kappa p} d \mu\right)^{1 / \kappa} d t \\
\leq & \frac{\mu\left(B_{j}\right)}{\mu\left(B_{j+1}\right)}\left(\underset{\Gamma_{j}}{\operatorname{ess} \sup } f_{B_{j}} u^{1+\varepsilon} \phi_{j}^{p} d \mu\right)^{(\kappa-1) / \kappa} \\
& \times \int_{\Gamma_{j}}\left(f_{B_{j}}\left(u^{(p-1+\varepsilon) / p} \phi_{j}\right)^{\kappa p} d \mu\right)^{1 / \kappa} d t
\end{aligned}
$$

where $\kappa$ is as in (2.1). The ratio of the measures of balls is bounded by the doubling constant $C_{\mu}$ of $\mu$. The Sobolev inequality together with the 
Caccioppoli estimate, Lemma 3.6, gives

$$
\begin{aligned}
& f_{Q_{j+1}} u^{(1+\varepsilon)(\kappa-1) / \kappa+p-1+\varepsilon} d \mu d t \\
& \leq C \frac{R^{p}}{T_{j}}\left(\underset{\Gamma_{j}}{\operatorname{esssup}} f_{B_{j}} u^{1+\varepsilon} \phi_{j}^{p} d \mu\right)^{(\kappa-1) / \kappa} \\
& \quad \times \int_{\Gamma_{j}} f_{B_{j}}\left|\nabla\left(u^{(p-1+\varepsilon) / p} \phi_{j}\right)\right|^{p} d \mu d t . \\
& \leq C \varepsilon^{p} R^{p} T_{j}^{1-1 / \kappa} \\
& \quad \times\left(f_{Q_{j}} u^{p-1+\varepsilon}|\nabla \phi|^{p}+u^{1+\varepsilon}\left(\frac{\partial \phi^{p}}{\partial t}\right)_{+} d \mu d t\right)^{2-1 / \kappa} .
\end{aligned}
$$

Note here that $\left|\Gamma_{j}\right| \geq T_{j} / 2$. The assumption $u^{2-p} \leq T / R^{p}$ implies

$$
\begin{aligned}
f_{Q_{j+1}} & u^{(1+\varepsilon)(\kappa-1) / \kappa+p-1+\varepsilon} d \mu d t \\
\leq & C \varepsilon^{2 p} R^{p} T_{j}^{1-1 / \kappa}\left(f_{Q_{j}} u^{p-1+\varepsilon}\left(|\nabla \phi|^{p}+\frac{T}{R^{p}}\left(\frac{\partial \phi^{p}}{\partial t}\right)_{+}\right) d \mu d t\right)^{2-1 / \kappa} \\
\leq & C \varepsilon^{2 p} 4^{j p}\left(\frac{T_{j}}{R^{p}}\right)^{1-1 / \kappa}\left(\frac{1}{(\rho-\sigma)^{p}} f_{Q_{j}} u^{p-1+\varepsilon} d \mu d t\right)^{2-1 / \kappa} .
\end{aligned}
$$

We then choose $\varepsilon=\varepsilon_{j}$, where $\varepsilon_{j}$ is a solution to the difference equation

$$
p-1+\varepsilon_{j+1}=\left(1+\varepsilon_{j}\right) \frac{\kappa-1}{\kappa}+p-1+\varepsilon_{j}, \quad \varepsilon_{0}=s, \quad s \geq 1 .
$$

Indeed, $\varepsilon_{j}=(1+s)(2-1 / \kappa)^{j}-1, j=0,1, \ldots$, solves the equation. We set $\alpha_{j}=p-1+\varepsilon_{j}$ and conclude

$$
f_{Q_{j+1}} u^{\alpha_{j+1}} d \mu d t \leq C 16^{p j}\left(\frac{T}{R^{p}}\right)^{1-1 / \kappa}\left(\frac{1}{(\rho-\sigma)^{p}} f_{Q_{j}} u^{\alpha_{j}} d \mu d t\right)^{2-1 / \kappa} .
$$

Iteration of the obtained inequality gives

$$
\begin{aligned}
& f_{Q_{j+1}} u^{\alpha_{j+1}} d \mu d t \\
& \leq 16^{p \sum_{i=0}^{j}(j-i)(2-1 / \kappa)^{i}}\left(\frac{C T}{R^{p}}\right)^{(1-1 / \kappa) \sum_{i=0}^{j}(2-1 / \kappa)^{i}} \\
& \quad \times\left(\frac{1}{\rho-\sigma}\right)^{p(2-1 / \kappa) \sum_{i=0}^{j}(2-1 / \kappa)^{i}}\left(f_{Q_{j}} u^{\alpha_{0}} d \mu d t\right)^{(2-1 / \kappa)^{j+1}} \\
& \leq \frac{C R^{p}}{T}\left(\frac{T}{R^{p}}\left(\frac{1}{\rho-\sigma}\right)^{p(2 \kappa-1) /(\kappa-1)} f_{Q_{j}} u^{\alpha_{0}} d \mu d t\right)^{(2-1 / \kappa)^{j+1}} .
\end{aligned}
$$

We take power $1 / \alpha_{j+1}$ from both sides, let $j \rightarrow \infty, h \rightarrow 0$, and obtain

$$
\underset{U_{\sigma}}{\operatorname{ess} \sup } u \leq\left(C \frac{T}{R^{p}}\left(\frac{1}{\rho-\sigma}\right)^{p(2 \kappa-1) /(\kappa-1)} f_{U_{\rho}} u^{p-1+s} d \mu d t\right)^{1 /(1+s)},
$$

where $U_{\sigma}=B\left(x_{0}, \sigma R\right) \times\left(t_{0}-\sigma^{p} T, t_{0}\right)$. The result follows then for $\delta \geq 2$. By the previous estimate with $s=1$ and Young's inequality, we obtain for 
every $2>\delta \geq \min \left\{\delta_{0}, 1\right\}$ that

$$
\begin{aligned}
\underset{U_{\sigma}}{\operatorname{ess} \sup u} & \leq\left(\underset{U_{\rho}}{\operatorname{ess} \sup } u^{2-\delta} C \frac{T}{R^{p}}\left(\frac{1}{\rho-\sigma}\right)^{p(2 \kappa-1) /(\kappa-1)} f_{U_{\rho}} u^{p-2+\delta} d \mu d t\right)^{1 / 2} \\
& \leq \frac{1}{2} \underset{U_{\rho}}{\operatorname{ess} \sup } u+\left(C \frac{T}{R^{p}}\left(\frac{1}{\rho-\sigma}\right)^{p(2 \kappa-1) /(\kappa-1)} f_{U_{\rho}} u^{p-2+\delta} d \mu d t\right)^{1 / \delta} .
\end{aligned}
$$

A standard iteration argument (see e.g. [22] Lemma 5.1) implies the assertion of the lemma.

We use the previous lemma to show the preliminary version of the first statement in Theorem 3.1.

Lemma 3.17. Let $u$ be a subsolution in $B\left(x_{0}, R\right) \times\left(t_{0}-T, t_{0}\right)$. Suppose that

$$
u^{p-2}(x, t) \geq \frac{R^{p}}{T} \quad \text { for all } \quad(x, t) \in B\left(x_{0}, R\right) \times\left(t_{0}-T, t_{0}\right) .
$$

Then there are constants $C_{i}=C_{i}\left(p, \alpha, \beta, C_{\mu}, C_{p}\right), i=1,2$, such that

$$
\underset{B\left(x_{0}, \sigma R\right) \times\left(t_{0}-\sigma^{p} T, t_{0}\right)}{\operatorname{ess} \sup _{R^{p}}} u \leq \frac{T}{(1-\sigma)^{C_{2}}}\left(\operatorname{ess~sup}_{t_{0}-T<t<t_{0}} f_{B\left(x_{0}, R\right)} u d \mu\right)^{p-1}
$$

for all $0<\sigma<1$.

Proof. Let $0<h<1 / 2$. We set

$$
U_{\sigma}=B\left(x_{0}, \sigma R\right) \times\left(t_{0}-\sigma^{p} T, t_{0}-h \sigma^{p} T\right),
$$

$0<\sigma<1$. We choose the test function $\phi \in C_{0}^{\infty}\left(U_{\rho}\right), 0<\sigma<\rho<1$, such that $0 \leq \phi \leq 1, \phi_{j}=1$ in $U_{\sigma}$ and

$$
|\nabla \phi| \leq \frac{C}{R(\rho-\sigma)}, \quad\left(\frac{\partial \phi^{p}}{\partial t}\right)_{+} \leq \frac{C}{T(\rho-\sigma)^{p}} .
$$

By Hölder's inequality, we obtain

$$
\begin{aligned}
& f_{U_{\sigma}} u^{p+(\kappa-1) / \kappa} d \mu d t \\
& \quad \leq C\left(\operatorname{ess~sup}_{t_{0}-T<t<t_{0}} f_{B\left(x_{0}, R\right)} u d \mu\right)^{(\kappa-1) / \kappa} f_{t_{0}-T}^{t_{0}}\left(f_{B\left(x_{0}, \rho R\right)}(u \phi)^{\kappa p} d \mu\right)^{1 / \kappa} d t .
\end{aligned}
$$

Similarly as in the proof of Lemma 3.15, from the Sobolev and Caccioppoli inequalities, it follows that

$$
\begin{aligned}
& f_{U_{\sigma}} u^{p+(\kappa-1) / \kappa} d \mu d t \\
& \quad \leq C R^{p}\left(\operatorname{esssup}_{t_{0}-T<t<t_{0}} f_{B\left(x_{0}, R\right)} u d \mu\right)^{(\kappa-1) / \kappa} f_{U_{\rho}}|\nabla(u \phi)|^{p} d \mu d t \\
& \leq \frac{C}{(\rho-\sigma)^{p}}\left(\underset{t_{0}-T<t<t_{0}}{\operatorname{ess} \sup _{B\left(x_{0}, R\right)}} f_{d \mu)^{(\kappa-1) / \kappa}} f_{U_{\rho}} u^{p} d \mu d t .\right.
\end{aligned}
$$


Here we also use the assumption $u^{2-p} \leq T / R^{p}$. Furthermore, by Hölder's and Young's inequalities, we get

$$
\begin{aligned}
& f_{U_{\sigma}} u^{p} d \mu d t \leq\left(f_{U_{\sigma}} u^{p+(\kappa-1) / \kappa} d \mu d t\right)^{\kappa p /(\kappa(p+1)-1)} \\
& \quad \leq\left(\frac{C}{(\rho-\sigma)^{p}}\left(\operatorname{ess}_{t_{0}-T<t<t_{0}} f_{B\left(x_{0}, R\right)} u d \mu\right)^{(\kappa-1) / \kappa} f_{U_{\rho}} u^{p} d \mu d t\right)^{\kappa p /(\kappa(p+1)-1)} \\
& \quad \leq \frac{1}{2} f_{U_{\rho}} u^{p} d \mu d t+\frac{C}{(\rho-\sigma)^{C}}\left(\operatorname{ess~sup}_{t_{0}-T<t<t_{0}} f_{B\left(x_{0}, R\right)} u d \mu\right)^{p}
\end{aligned}
$$

for all $0<\sigma<\rho<1$. The iteration then gives

$$
f_{U_{\sigma}} u^{p} d \mu d t \leq \frac{C}{(1-\sigma)^{C}}\left(\operatorname{esssup}_{t_{0}-T<t<t_{0}} f_{B\left(x_{0}, R\right)} u d \mu\right)^{p}
$$

for all $0<\sigma<1$. We now use Lemma 3.15 together with Hölder's inequality and arrive at

$$
\begin{aligned}
\underset{U_{\sigma}}{\operatorname{ess} \sup u} & \leq \frac{T}{R^{p}} \frac{C}{(1-\sigma)^{C}} f_{U_{(1+\sigma) / 2}} u^{p-1} d \mu d t \\
& \leq \frac{T}{R^{p}} \frac{C}{(1-\sigma)^{C}}\left(f_{U_{(1+\sigma) / 2}} u^{p} d \mu d t\right)^{(p-1) / p} \\
& \leq \frac{T}{R^{p}} \frac{C}{(1-\sigma)^{C}}\left(\operatorname{tass}_{t_{0}-T<t<t_{0}} f_{B\left(x_{0}, R\right)} u d \mu\right)^{p-1},
\end{aligned}
$$

which proves the result after letting $h \rightarrow 0$.

An application of the previous lemma for $v=u+\left(R^{p} / T\right)^{1 /(p-2)}$ gives the first statement in Theorem 3.1. Furthermore, the result implies the following corollary, which will be used in the proof of the gradient estimate, the third statement in Theorem 3.1. Note carefully that we do not use the scaling argument in the proof. The customary way to obtain the same result is to blow-up the solution near the initial instant, see for example Choe-Lee [14]. The advantage of our proof is its local nature, and, in particular, we apply only the local Sobolev inequality and doubling condition.

Corollary 3.18. Let $u$ be a non-negative subsolution in $B\left(x_{0}, R\right) \times\left(t_{0}-\right.$ $\left.T, t_{0}\right)$. Suppose further that

$$
\rho=\left(R^{d_{\mu}} \operatorname{esssup}_{t_{0}-T<t<t_{0}} f_{B\left(x_{0}, R\right)} u d \mu\right)^{(p-2) / \lambda} T^{1 / \lambda} \leq R,
$$

where $\lambda=d_{\mu}(p-2)+p$. Then there is a constant $C=C\left(p, \alpha, \beta, C_{\mu}, C_{p}\right)$ such that

$$
\underset{Q}{\operatorname{ess} \sup } u \leq C T^{-d_{\mu} / \lambda}\left(R^{d_{\mu}} \underset{t_{0}-T<t<t_{0}}{\operatorname{ess} \sup _{B\left(x_{0}, R\right)}} u d \mu\right)^{p / \lambda},
$$

where $Q=B\left(x_{0}, \rho / 2\right) \times\left(t_{0}-T / 2, t_{0}\right)$.

Proof. We apply the first statement in Theorem 3.1 with $\rho / 2$ instead of $R$ and obtain

$$
\underset{Q}{\operatorname{ess} \sup } u \leq C\left(\frac{\rho^{p}}{T}\right)^{1 /(p-2)}+C \frac{T}{\rho^{p}}\left(\operatorname{ess}_{t_{0}-T<t<t_{0}}^{\operatorname{esup}} f_{B\left(x_{0}, \rho\right)} u d \mu\right)^{p-1} .
$$


The doubling condition implies

$$
\mu\left(B\left(x_{0}, R\right)\right) \leq C_{\mu}\left(\frac{R}{\rho}\right)^{d_{\mu}} \mu\left(B\left(x_{0}, \rho\right)\right) .
$$

Hence we get

$$
\operatorname{esssup}_{t_{0}-T<t<t_{0}} f_{B\left(x_{0}, \rho\right)} u d \mu \leq C_{\mu}\left(\frac{R}{\rho}\right)^{d_{\mu}} \underset{t_{0}-T<t<t_{0}}{\operatorname{esssup}} f_{B\left(x_{0}, R\right)} u d \mu,
$$

and, consequently,

$$
\underset{Q}{\operatorname{ess} \sup } u \leq C\left(\frac{\rho^{p}}{T}\right)^{1 /(p-2)}+C \frac{T}{\rho^{\lambda+d_{\mu}}}\left(R^{d_{\mu}} \operatorname{ess~sup}_{t_{0}-T<t<t_{0}} f_{B\left(x_{0}, R\right)} u d \mu\right)^{p-1} .
$$

The result follows by inserting the definition of $\rho$ into the inequality above.

Furthermore, in view of Remark 3.10, if the subsolution has zero initial data, one may repeat the proofs of Lemmas 3.15 and 3.17 using the test functions depending only on the spatial variable $x$. Hence the positivity assumption $u^{p-2} \geq R^{p} / T$ may be removed.

Lemma 3.19. Let $u$ be a non-negative subsolution in $B\left(x_{0}, R\right) \times\left(t_{0}, t_{0}+T\right)$. Suppose further that

$$
\lim _{h \rightarrow 0} \int_{t_{0}+h}^{t_{0}+2 h} \int_{B\left(x_{0}, R\right)} u^{2} d \mu d t=0 .
$$

Then there are constants $C_{i}=C_{i}\left(p, \alpha, \beta, C_{\mu}, C_{p}, \delta_{0}\right), i=1,2, \delta_{0}>0$, such that

$$
\operatorname{ess~sup}_{B\left(x_{0}, \sigma R\right) \times\left(t_{0}, t_{0}+T\right)} u \leq\left(\frac{C_{1}}{(1-\sigma)^{C_{2}}} \frac{T}{R^{p}} f_{t_{0}}^{t_{0}+T} f_{B\left(x_{0}, R\right)} u^{p-2+\delta} d \mu d t\right)^{1 / \delta},
$$

$\delta \geq \delta_{0}$, and

$$
\operatorname{ess~sup}_{B\left(x_{0}, \sigma R\right) \times\left(t_{0}, t_{0}+T\right)} u \leq \frac{C_{1}}{(1-\sigma)^{C_{2}}} \frac{T}{R^{p}}\left(\operatorname{ess~sup}_{t_{0}<t<t_{0}+T} f_{B\left(x_{0}, R\right)} u d \mu\right)^{p-1}
$$

for all $0<\sigma<1$.

We apply the result to prove the second statement in Theorem 3.1.

Proof of Theorem 3.1 ii). We set $R_{j}=\left(2^{-j-1}+1\right) R / 2$ and denote

$$
\Upsilon_{j}=\operatorname{ess}_{B\left(x_{0}, R_{j}\right) \times\left(t_{0}, t_{0}+\tau\right)} u \text {. }
$$

On the one hand, we have from Lemma 3.19 that

$$
\begin{aligned}
\Upsilon_{j+1} & \leq C^{j} \frac{\tau}{R^{p}} f_{t_{0}}^{t_{0}+\tau} f_{B\left(x_{0}, R_{j}\right)} u^{p-1} d \mu d t \\
& \leq C^{j} \frac{\tau}{R^{p}} \Upsilon_{j}^{1+p-2} .
\end{aligned}
$$

An iteration argument (see e.g. [17], Lemma 4.1, p. 12) yields that $\Upsilon_{j} \rightarrow 0$ as $j \rightarrow \infty$, if

$$
\Upsilon_{0} \leq\left(\frac{\tau}{R^{p}}\right)^{-1 /(p-2)} C^{-1 /(p-2)^{2}}
$$


On the other hand, the second statement in Lemma 3.19 gives

$$
\Upsilon_{0} \leq C \frac{\tau}{R^{p}}\left(\underset{t_{0}<t<t_{0}+\tau}{\operatorname{ess} f_{B\left(x_{0}, R\right)}} u d \mu\right)^{p-1} .
$$

The right hand side of (3.21) is smaller than the right hand side of (3.20) due to the condition (3.3). This finishes the proof.

We finally prove the estimate for the gradient. Similar proof for solutions can be found in [17] and in the global setting in [14]. The main ingredient of the proof is Corollary 3.18. Notice that due to the local nature of the corollary, also the gradient estimate is local.

Proof of Theorem 3.1 iii). Without losing the generality, we may assume that $t_{0}=0$. Recall that by the assumption, we have

$$
\tau \leq R^{p} N_{\tau}^{2-p}=R^{p}\left(\underset{0<t<\tau}{\operatorname{esssup}} f_{B\left(x_{0}, 2 R\right)} u d \mu\right)^{2-p} .
$$

First, we use Hölder's inequality and get

$$
\begin{aligned}
& \int_{0}^{\tau} f_{B\left(x_{0}, R\right)}|\nabla u|^{p-1} d \mu d t \\
& =\int_{0}^{\tau} f_{B\left(x_{0}, R\right)}|\nabla u|^{p-1} u^{1 / 2} u^{-1 / 2} t^{1 /(2 p)} t^{-1 /(2 p)} d \mu d t \\
& \leq\left(\int_{0}^{\tau} f_{B\left(x_{0}, R\right)} u^{p / 2} t^{-1 / 2} d \mu d t\right)^{1 / p} \\
& \quad \times\left(\int_{0}^{\tau} f_{B\left(x_{0}, R\right)} u^{-p /(2 p-2)} t^{1 /(2 p-2)}|\nabla u|^{p} d \mu d t\right)^{(p-1) / p}
\end{aligned}
$$

Since (3.22) implies that

$$
t^{1 / \lambda}\left(R^{d_{\mu}} N_{t}\right)^{(p-2) / \lambda} \leq \tau^{1 / \lambda}\left(R^{d_{\mu}} N_{\tau}\right)^{(p-2) / \lambda} \leq R,
$$

we have by Corollary 3.18 and the doubling condition that

$$
\operatorname{ess~sup}_{B\left(x_{0}, 3 R / 2\right) \times(t / 2, t)} u \leq C t^{-d_{\mu} / \lambda}\left(R^{d_{\mu}} N_{t}\right)^{p / \lambda} \leq C t^{-d_{\mu} / \lambda}\left(R^{d_{\mu}} N_{\tau}\right)^{p / \lambda}
$$

for every $0<t \leq \tau$. This implies that

$$
\begin{aligned}
\Psi_{1}(\tau) & :=\int_{0}^{\tau} f_{B\left(x_{0}, R\right)} u^{p / 2} t^{-1 / 2} d \mu d t \\
& \leq \int_{0}^{\tau} t^{-1 / 2}\|u\|_{L^{\infty}\left(B\left(x_{0}, R\right) \times(t / 2, t)\right)}^{p-1} f_{B\left(x_{0}, R\right)} u d \mu d t \\
& \leq C N_{\tau}\left(R^{d_{\mu}} N_{\tau}\right)^{p(p-2) /(2 \lambda)} \int_{0}^{\tau} t^{-1 / 2-d_{\mu}(p-2) /(2 \lambda)} d t \\
& =C N_{\tau}\left(R^{d_{\mu}} N_{\tau}\right)^{p(p-2) /(2 \lambda)} \tau^{p /(2 \lambda)} .
\end{aligned}
$$

Next, we choose

$$
\varepsilon=\frac{p-2}{2 p-2}, \quad \phi=t^{1 /(p(2 p-2))} \theta
$$


where $\theta \in C_{0}^{\infty}\left(B\left(x_{0}, 3 R / 2\right)\right)$ depends only on the spatial variable, $0 \leq \theta \leq 1$, $\theta=1$ in $B\left(x_{0}, R\right)$, and $|\nabla \theta| \leq C / R$. By Remark 3.9 , we may substitute the choices into (3.8) and obtain

$$
\begin{aligned}
\Psi_{2}(\tau):= & \int_{0}^{\tau} f_{B\left(x_{0}, R\right)}|\nabla u|^{p} u^{-1+(p-2) /(2 p-2)} t^{1 /(2 p-2)} d \mu d t \\
\leq & C\left(\frac{p-1}{p-2}\right)^{p} \int_{0}^{\tau} f_{B\left(x_{0}, 3 R / 2\right)} u^{p-1+(p-2) /(2 p-2)} t^{1 /(2 p-2)}|\nabla \theta|^{p} d \mu d t \\
& +C \frac{p-1}{p-2} \int_{0}^{\tau} f_{B\left(x_{0}, 3 R / 2\right)} u^{1+(p-2) /(2 p-2)} t^{-1+1 /(2 p-2)} d \mu d t \\
& -C \frac{p-1}{p-2} f_{B\left(x_{0}, 3 R / 2\right)} u^{1+(p-2) /(2 p-2)}(x, \tau) \tau^{1 /(2 p-2)} \theta^{p}(x) d \mu(x) .
\end{aligned}
$$

Here we have also used the doubling condition. We further estimate

$$
\begin{aligned}
\Psi_{2}(\tau) \leq & \frac{C}{R^{p}} \int_{0}^{\tau} f_{B\left(x_{0}, 3 R / 2\right)} u^{p-1+(p-2) /(2 p-2)} t^{1 /(2 p-2)} d \mu d t \\
& +C \int_{0}^{\tau} f_{B\left(x_{0}, 3 R / 2\right)} u^{1+(p-2) /(2 p-2)} t^{-1+1 /(2 p-2)} d \mu d t .
\end{aligned}
$$

For the first term on the right hand side, we have

$$
\begin{aligned}
& \int_{0}^{\tau} f_{B\left(x_{0}, 3 R / 2\right)} u^{p-1+(p-2) /(2 p-2)} t^{1 /(2 p-2)} d \mu d t \\
& \leq \int_{0}^{\tau}\|u\|_{L^{\infty}\left(B\left(x_{0}, 3 R / 2\right) \times(t / 2, t)\right)}^{(p-2)(1+1 /(2 p-2))} t^{1 /(2 p-2)} f_{B\left(x_{0}, 3 R / 2\right)} u d \mu d t \\
& \leq C N_{\tau}\left(R^{d_{\mu}} N_{\tau}\right)^{(p / \lambda)(p-2)(1+1 /(2 p-2))} \int_{0}^{\tau} t^{-\left(d_{\mu}(p-2) / \lambda\right)(1+1 /(2 p-2))+1 /(2 p-2)} d t \\
& =C N_{\tau}\left(R^{d_{\mu}} N_{\tau}\right)^{(p / \lambda)(p-2)(1+1 /(2 p-2))} \tau^{p(2 p-1) /(\lambda(2 p-2))} \\
& =C N_{\tau}\left(\left(R^{d_{\mu}} N_{\tau}\right)^{p-2} \tau\right)^{p / \lambda}\left(R^{d_{\mu}} N_{\tau}\right)^{(p / \lambda)(p-2) /(2 p-2)} \tau^{p /(\lambda(2 p-2))} \\
& \leq C N_{\tau} R^{p}\left(R^{d_{\mu}} N_{\tau}\right)^{(p / \lambda)(p-2) /(2 p-2)} \tau^{p /(\lambda(2 p-2))},
\end{aligned}
$$

where the last inequality follows by (3.23). Similarly, the second term can be estimated as

$$
\begin{aligned}
& \int_{0}^{\tau} f_{B\left(x_{0}, 3 R / 2\right)} u^{1+(p-2) /(2 p-2)} t^{-1+1 /(2 p-2)} d \mu d t \\
& \leq C N_{\tau}\left(R^{d_{\mu}} N_{\tau}\right)^{(p / \lambda)(p-2) /(2 p-2)} \int_{0}^{\tau} t^{-\left(d_{\mu} / \lambda\right)(p-2) /(2 p-2)-1+1 /(2 p-2)} d t \\
& =C N_{\tau}\left(R^{d_{\mu}} N_{\tau}\right)^{(p / \lambda)(p-2) /(2 p-2)} \tau^{p /(\lambda(2 p-2))} .
\end{aligned}
$$

Combining the estimates, we arrive at

$$
\Psi_{2}(\tau) \leq C N_{\tau}\left(R^{d_{\mu}} N_{\tau}\right)^{(p / \lambda)(p-2) /(2 p-2)} \tau^{p /(\lambda(2 p-2))} .
$$

Thus we conclude that

$$
\begin{aligned}
\int_{0}^{\tau} f_{B\left(x_{0}, R\right)}|\nabla u|^{p-1} d \mu d t & \leq \Psi_{1}(\tau)^{1 / p} \Psi_{2}(\tau)^{(p-1) / p} \\
& \leq C\left(\left(R^{d_{\mu}} N_{\tau}\right)^{p-2} \tau\right)^{1 / \lambda} N_{\tau}
\end{aligned}
$$


which proves the claim.

3.1. Zero lateral boundary data. We yet complement the results of the previous section. In particular, we show that every subsolution to the Dirichlet boundary value problem with zero lateral boundary data is bounded above by a constant independent of the initial data.

Lemma 3.24. Let $u$ be a non-negative subsolution in $\Omega \times\left(t_{0}-T, t_{0}\right)$, where $\Omega$ is a bounded, and $R=\operatorname{diam}(\Omega)<\infty$. Suppose that

$$
u \in L^{p}\left(t_{0}-T, t_{0} ; W_{0}^{1, p}(\Omega)\right)
$$

Then there exists a constant $C=C\left(p, \alpha, \beta, C_{\mu}, C_{p}\right)$ such that for all $\delta \geq 0$, we have

$$
\underset{Q}{\operatorname{ess} \sup } u \leq\left(C\left(\frac{R^{p}}{T}\right)^{\kappa /(\kappa-1)} f_{t_{0}-T}^{t_{0}} f_{\Omega} u^{\delta} d \mu\right)^{1 /(\delta+(p-2) \kappa /(\kappa-1)},
$$

where $Q=\Omega \times\left(t_{0}-T / 2, t_{0}\right)$.

Proof. We set $T_{j}=\sigma T+(\rho-\sigma) 2^{-j} T, 0<\sigma<\rho<1$, and

$$
\Gamma_{j}=\left(t_{0}-T_{j}, t_{0}-h T_{j}\right), \quad Q_{j}=\Omega \times \Gamma_{j},
$$

where $0<h<\sigma / 2$. We choose test functions $\phi_{j} \in C_{0}^{\infty}\left(\Gamma_{j}\right)$ such that $0 \leq \phi_{j} \leq 1, \phi_{j}=1$ in $\Gamma_{j+1}$, and

$$
\left(\frac{\partial \phi^{p}}{\partial t}\right)_{+} \leq \frac{C 2^{j}}{T(\rho-\sigma)}
$$

Furthermore, let $\varepsilon \geq 1$. Due to the fact that $u$ has zero lateral boundary values in Sobolev sense, it has zero extension outside of $\Omega$. Hence, by Remark 3.11 , we may insert $\phi_{j}$ into (3.16), and obtain

$$
\begin{aligned}
& f_{Q_{j+1}} u^{(1+\varepsilon)(\kappa-1) / \kappa+p-1+\varepsilon} d \mu d t \\
& \leq C \varepsilon^{2 p} 4^{j p} \frac{R^{p}}{T}\left(\frac{1}{\rho-\sigma} f_{Q_{j}} u^{1+\varepsilon} d \mu d t\right)^{2-1 / \kappa} .
\end{aligned}
$$

Observe that we apply the Sobolev inequality in a ball with the radius $R$. Let $\varepsilon_{j}$ be a solution to the difference equation

$$
1+\varepsilon_{j+1}=\left(1+\varepsilon_{j}\right) \frac{\kappa-1}{\kappa}+p-1+\varepsilon_{j}, \quad \varepsilon_{0}=s, \quad s \geq 1 .
$$

The equation has the solution

$$
\varepsilon_{j}=\left(s+1+\frac{\kappa(p-2)}{\kappa-1}\right)\left(2-\frac{1}{\kappa}\right)^{j}-\frac{\kappa(p-2)}{\kappa-1}-1 .
$$

We set $\alpha_{j}=1+\varepsilon_{j}$ and conclude

$$
\begin{aligned}
f_{Q_{j+1}} & u^{\alpha_{j+1}} d \mu d t \leq\left(\frac{R^{p}}{T}\right)^{\kappa /(\kappa-1)} \\
& \times\left(\left(\frac{C R^{p}}{T}\right)^{\kappa /(\kappa-1)}\left(\frac{1}{\rho-\sigma}\right)^{(2 \kappa-1) /(\kappa-1)} f_{Q_{j}} u^{\alpha_{0}} d \mu d t\right)^{(2-1 / \kappa)^{j+1}} .
\end{aligned}
$$


Taking power $1 / \alpha_{j+1}$ from both sides and letting $j \rightarrow \infty, h \rightarrow 0$, implies

$$
\underset{U_{\sigma}}{\operatorname{ess} \sup } u \leq\left(C\left(\frac{R^{p}}{T}\right)^{\kappa /(\kappa-1)}\left(\frac{1}{\rho-\sigma}\right)^{C} f_{U_{\rho}} u^{1+s} d \mu d t\right)^{1 /(1+s+(p-2) \kappa /(\kappa-1))},
$$

where $U_{\sigma}=B\left(x_{0}, R\right) \times\left(t_{0}-\sigma^{p} T, t_{0}\right)$. This proves the result for $\delta \geq 2$. For $0 \leq \delta<2$, we obtain by the previous estimate with $s=1$ that

$$
\begin{aligned}
\underset{U_{\sigma}}{\operatorname{ess} \sup u \leq} & \left(\underset{U_{\rho}}{\operatorname{ess} \sup } u^{2-\delta}\right. \\
& \left.\times C\left(\frac{R^{p}}{T}\right)^{\kappa /(\kappa-1)}\left(\frac{1}{\rho-\sigma}\right)^{C} \int_{U_{\rho}} u^{\delta} d \mu d t\right)^{1 /(2+(p-2) \kappa /(\kappa-1))} \\
\leq & \frac{1}{2} \operatorname{ess}_{U_{\rho}} u \\
& +\left(\left(\frac{R^{p}}{T}\right)^{\kappa /(\kappa-1)}\left(\frac{C}{\rho-\sigma}\right)^{C} \int_{U_{\rho}} u^{\delta} d \mu d t\right)^{1 /(\delta+(p-2) \kappa /(\kappa-1))}
\end{aligned}
$$

and the result follows by the iteration.

In the degenerate case the diffusion is very fast, whenever the gradient is large. This fact leads to a uniform estimate for the weak solution in the case of zero lateral boundary values after a waiting time: simply choose $\delta=0$ in the previous lemma. Notice that the estimate is independent of the initial data. Intuitively, with large initial data, the diffusion is faster and the solution decreases more rapidly. Notice that this is not true for the linear heat equation since the multiples of solutions remain solutions.

Corollary 3.25. Let $u$ be a non-negative subsolution in $\Omega \times\left(t_{0}-T, t_{0}\right)$, where $\Omega$ is an open set and $R=\operatorname{diam}(\Omega)<\infty$. Suppose further that

$$
u \in L_{l o c}^{p}\left(t_{0}-T, t_{0} ; W_{0}^{1, p}(\Omega)\right) .
$$

Then there exists a constant $C=C\left(p, \alpha, \beta, C_{\mu}, C_{p}\right)$ such that

$$
\operatorname{esssup}_{B\left(x_{0}, R\right) \times\left(t_{0}-T / 2, t_{0}\right)} u \leq C\left(\frac{R^{p}}{T}\right)^{1 /(p-2)} .
$$

\section{Compactness for solutions}

In this section, we show that every bounded sequence of solutions has a subsequence converging to a solution. This result has a central role in the proof of the main result. The compactness result is proved by first applying parabolic counterpart of Rellich's theorem to obtain strong convergence for solutions and weak convergence for gradients. However, the passage to the limit in the weak formulation requires much more information than the weak convergence of the gradient. We establish the essential pointwise convergence of the gradient by applying the monotonicity of the equation via Cauchy sequences and a priori estimates.

Lemma 4.1. Let $u_{i}, i=1,2, \ldots$ be a sequence of uniformly essentially bounded weak solutions in $M_{\tau_{1}, \tau_{2}}=M \times\left(\tau_{1}, \tau_{2}\right)$. Then the sequence $\nabla u_{i}, i=$ 
$1,2, \ldots$ is locally uniformly bounded in $L^{p}\left(M_{\tau_{1}, \tau_{2}}\right)$. Furthermore, there exists a subsequence still denoted by $u_{i}, i=1,2, \ldots$ such that

$$
\begin{aligned}
u_{i} & \rightarrow u \quad \text { in } \quad L_{l o c}^{p}\left(M_{\tau_{1}, \tau_{2}}\right) \quad \text { and } \\
\nabla u_{i} & \rightarrow \nabla u \quad \text { weakly in } L_{l o c}^{p}\left(M_{\tau_{1}, \tau_{2}}\right) .
\end{aligned}
$$

Proof. First, we show that the sequence $u_{i}, i=1,2, \ldots$, is bounded in $L^{p}\left(t_{1}, t_{2} ; W^{1, p}\left(\Omega^{\prime}\right)\right)$, where $\Omega_{t_{1}, t_{2}}^{\prime}=\Omega^{\prime} \times\left(t_{1}, t_{2}\right) \Subset M_{\tau_{1}, \tau_{2}}$, and that the distributional time derivatives of $u_{i}$ are bounded in the dual space of $L^{p}\left(t_{1}, t_{2} ; W_{0}^{1, p}\left(\Omega^{\prime}\right)\right)$. Then we use standard compactness theorems.

To begin with, observe that by the Caccioppoli inequality, cf. Lemma 3.6, the sequence $\nabla u_{i}, i=1,2, \ldots$, is locally uniformly bounded in $M_{\tau_{1}, \tau_{2}}$. In addition, this fact, the definition of a weak solution, and the growth bounds of $\mathcal{A}$ imply

$$
\begin{aligned}
\left|\int_{\Omega_{t_{1}, t_{2}}^{\prime}} \frac{\partial \phi}{\partial t} u_{i} d \mu d t\right| & \leq C\left(\int_{\Omega_{t_{1}, t_{2}}^{\prime}}\left|\nabla u_{i}\right|^{p} d \mu d t\right)^{(p-1) / p}\left(\int_{\Omega_{t_{1}, t_{2}}^{\prime}}|\nabla \phi|^{p} d \mu d t\right)^{1 / p} \\
& \leq C\|\phi\|_{L^{p}\left(t_{1}, t_{2} ; W_{0}^{1, p}\left(\Omega^{\prime}\right)\right)},
\end{aligned}
$$

for any $\phi \in C^{\infty}\left(\Omega_{t_{1}, t_{2}}^{\prime}\right) \cap L^{p}\left(t_{1}, t_{2} ; W_{0}^{1, p}\left(\Omega^{\prime}\right)\right)$. By the density of smooth functions, the time derivative of $u_{i}$ is bounded in the dual space of $L^{p}\left(t_{1}, t_{2} ; W_{0}^{1, p}\left(\Omega^{\prime}\right)\right)$.

Now, according to the parabolic version of Rellich's theorem (Lions-Aubin theorem), see for example page 106 of Showalter [35], Boccardo et. al. [10], and Simon [36], there exists a subsequence, still denoted by $u_{i}, i=1,2, \ldots$, satisfying (4.2).

Theorem 4.3. Let $u$, and $u_{i}, i=1,2, \ldots$ be as in Lemma 4.1. Then $u$ is a weak solution in $M_{\tau_{1}, \tau_{2}}$.

Proof. Passing to a subsequence, if necessary, Lemma 4.1 provides the local weak convergence for the gradients and the local $L^{p}$-convergence for the weak supersolutions. It remains to establish a pointwise convergence almost everywhere for the gradients, since together with the continuity of $\mathcal{A}$ this allows us to pass to limit under the integral sign. To this end, choose $u_{j}$ and $u_{k}$ from the subsequence. Both $u_{j}$ and $u_{k}$ are weak solutions, and hence we obtain, by subtracting the equations, that

$$
\begin{aligned}
& -\int_{M \times\left(\tau_{1}, \tau_{2}\right)}\left(u_{j}-u_{k}\right) \frac{\partial \phi}{\partial t} d \mu d t \\
& \quad+\int_{M \times\left(\tau_{1}, \tau_{2}\right)}\left\langle\mathcal{A}\left(x, t, \nabla u_{j}\right)-\mathcal{A}\left(x, t, \nabla u_{k}\right), \nabla \phi\right\rangle d \mu d t=0 .
\end{aligned}
$$

Next let $\theta \in C_{0}^{\infty}\left(M_{\tau_{1}, \tau_{2}}\right)$ and choose formally the test function

$$
\phi=\theta(x, t)\left(u_{j}(x, t)-u_{k}(x, t)\right) .
$$


By inserting it into (4.4), we obtain

$$
\begin{aligned}
\int_{M_{\tau_{1}, \tau_{2}}} & \left\langle\mathcal{A}\left(x, t, \nabla u_{j}\right)-\mathcal{A}\left(x, t, \nabla u_{k}\right),\left(\nabla u_{j}-\nabla u_{k}\right) \theta\right\rangle d \mu d t \\
\leq & -\int_{M_{\tau_{1}, \tau_{2}}}\left\langle\mathcal{A}\left(x, t, \nabla u_{j}\right)-\mathcal{A}\left(x, t, \nabla u_{k}\right),\left(u_{j}-u_{k}\right) \nabla \theta\right\rangle d \mu d t \\
& +\int_{M_{\tau_{1}, \tau_{2}}}\left(u_{j}-u_{k}\right) \frac{\partial\left(\theta\left(u_{j}-u_{k}\right)\right)}{\partial t} d \mu d t .
\end{aligned}
$$

To justify the reasoning, the estimate should be free of the time derivatives of $u_{j}$ and $u_{k}$, and therefore, we integrate by parts and end up with

$$
\int_{M_{\tau_{1}, \tau_{2}}}\left(u_{j}-u_{k}\right) \frac{\partial\left(\theta\left(u_{j}-u_{k}\right)\right.}{\partial t} d \mu d t=\frac{1}{2} \int_{M_{\tau_{1}, \tau_{2}}}\left(u_{j}-u_{k}\right)^{2} \frac{\partial \theta}{\partial t} d \mu d t .
$$

Furthermore, Hölder's inequality, the growth bounds, and the fact that the sequence $\nabla u_{i}, i=1,2, \ldots$, is locally uniformly bounded in $L^{p}\left(M_{\tau_{1}, \tau_{2}}\right)$, imply

$$
\begin{gathered}
\int_{M_{\tau_{1}, \tau_{2}}}\left\langle\mathcal{A}\left(x, t, \nabla u_{j}\right)-\mathcal{A}\left(x, t, \nabla u_{k}\right),\left(u_{j}-u_{k}\right)_{h} \nabla \theta\right\rangle d \mu d t \\
\leq C\left(\int_{M_{\tau_{1}, \tau_{2}}}\left|u_{j}-u_{k}\right|^{p}|\nabla \theta|^{p} d \mu d t\right)^{1 / p} .
\end{gathered}
$$

We combine the estimates and deduce

$$
\begin{aligned}
\int_{M_{\tau_{1}, \tau_{2}}} & \left\langle\left(\mathcal{A}\left(x, t, \nabla u_{j}\right)-\mathcal{A}\left(x, t, \nabla u_{j}\right)\right),\left(\nabla u_{j}-\nabla u_{k}\right) \theta\right\rangle d \mu d t \\
\leq & C \int_{M_{\tau_{1}, \tau_{2}}}\left|\frac{\partial \theta}{\partial t}\right|\left|u_{j}-u_{k}\right|^{2} d \mu d t \\
& +C\left(\int_{M_{\tau_{1}, \tau_{2}}}\left|u_{j}-u_{k}\right|^{p}|\nabla \theta|^{p} d \mu d t\right)^{1 / p} .
\end{aligned}
$$

Since $u_{i}, i=1,2, \ldots$, converges in $L_{\mathrm{loc}}^{p}\left(M_{\tau_{1}, \tau_{2}}\right)$, it follows that the right hand side can be made as small as we wish.

The rest of the proof is rather standard, see, for example, BoccardoGallouët [11], and also [28]. For the convenience of the reader, we repeat the proof. We shall show that (4.5) implies, passing to a subsequence, if necessary, that $\nabla u_{i}, i=1,2, \ldots$ is a Cauchy sequence in convergence in measure, that is, for any $\Omega_{t_{1}, t_{2}}^{\prime}=\Omega^{\prime} \times\left(t_{1}, t_{2}\right) \Subset M_{\tau_{1}, \tau_{2}}$ and for all $\delta, \eta>0$ we can choose large enough $j$ and $k$ so that

$$
\left|\left\{(x, t) \in \Omega_{t_{1}, t_{2}}^{\prime}:\left|\nabla u_{j}-\nabla u_{k}\right| \geq \delta\right\}\right|<\eta .
$$

Here $|\cdot|$ stands for the measure of the space-time set. Together with the fact that the norms of the gradients are locally uniformly bounded this implies the pointwise convergence almost everywhere. 
We define the following sets

$$
\begin{aligned}
& E_{j k}=\left\{(x, t) \in \Omega_{t_{1}, t_{2}}^{\prime}:\left|\nabla u_{j}(x, t)-\nabla u_{k}(x, t)\right| \geq \rho\right\}, \\
& U_{j k}=\left\{(x, t) \in \Omega_{t_{1}, t_{2}}^{\prime}:\left|u_{j}(x, t)-u_{k}(x, t)\right| \leq \delta\right\}, \quad \complement U_{j k}=\Omega_{t_{1}, t_{2}}^{\prime} \backslash U_{j k}, \\
& V_{j k}=\left\{(x, t) \in \Omega_{t_{1}, t_{2}}^{\prime}:\left|\nabla u_{j}(x, t)\right|,\left|\nabla u_{k}(x, t)\right| \leq \lambda\right\}, \quad \complement V_{j k}=\Omega_{t_{1}, t_{2}}^{\prime} \backslash V_{j k} .
\end{aligned}
$$

First, we observe that

$$
\left|E_{j k}\right| \leq\left|E_{j k} \cap U_{j k}\right|+\left|\complement U_{j k}\right|,
$$

and, hence, it is enough to show that the measure of the sets on the right can be made smaller than any $\eta>0$. We further estimate

$$
\left|E_{j k} \cap U_{j k}\right| \leq\left|E_{j k} \cap U_{j k} \cap V_{j k}\right|+\left|\complement V_{j k}\right| .
$$

Note that the sequence $u_{i}, i=1,2, \ldots$, converges in measure due to the strong convergence. It follows that

$$
\left|\complement U_{j k}\right|<\frac{\eta}{3}
$$

for $j$ and $k$ large enough, and since the $L^{p}$-norms of the gradients are bounded, there exists $\lambda>0$ such that

$$
\left|\complement V_{j k}\right| \leq \frac{\eta}{3}
$$

Consequently, it is enough to concentrate on the measure of the set $E_{j k} \cap$ $U_{j k} \cap V_{j k}$. We define

$$
\gamma(x, t)=\inf \langle\mathcal{A}(x, t, \xi)-\mathcal{A}(x, t, \zeta), \xi-\zeta\rangle,
$$

where the infimum is taken over the compact set

$$
\left\{(\xi, \zeta) \in T_{x} M \times T_{x} M:|\xi|,|\zeta| \leq \lambda,|\mathcal{A}(x, t, \xi)-\mathcal{A}(x, t, \zeta)| \geq \rho\right\} .
$$

Due to the continuity of $\xi \mapsto \mathcal{A}(x, t, \xi)$, the above set is compact. Hence the monotonicity of $\mathcal{A}$ implies that $\gamma(x, t)>0$ for almost every $(x, t) \in \Omega^{\prime}{ }_{t_{1}, t_{2}}$. According to (4.5), we obtain by choosing $\theta=1$ in $\Omega_{t_{1}, t_{2}}^{\prime}=\Omega^{\prime} \times\left(t_{1}, t_{2}\right)$ that for every $\delta>0$,

$$
\begin{aligned}
& \int_{E_{j k} \cap U_{j k} \cap V_{j k}} \gamma(x, t) d \mu d t \\
& \quad \leq \int_{U_{j k}}\left\langle\mathcal{A}\left(x, t, \nabla u_{j}\right)-\mathcal{A}\left(x, t, \nabla u_{k}\right), \nabla\left(u_{j}-u_{k}\right)\right\rangle d \mu d t \leq C \delta
\end{aligned}
$$

for $j$ and $k$ large enough. Since $\gamma(x, t)>0$ for almost every $(x, t) \in \Omega^{\prime}{ }_{t_{1}, t_{2}}$, it follows that

$$
\left|E_{j k} \cap U_{j k} \cap V_{j k}\right| \leq \frac{\eta}{3}
$$

for $\delta$ small enough and $j, k$ large enough. Combining the facts, we deduce

$$
\left|\left\{(x, t) \in \Omega^{\prime}{ }_{t_{1}, t_{2}}:\left|\nabla u_{j}(x, t)-\nabla u_{k}(x, t)\right| \geq \rho\right\}\right| \leq \eta
$$

for large enough $j$ and $k$.

Since the sequence $u_{i}, i=1,2, \ldots$ is bounded, converges to $u$, and $\nabla u_{i}$, $i=1,2, \ldots$, is a Cauchy sequence in convergence in measure, it follows that $\nabla u_{i}$ converges to $\nabla u$ in measure in $\Omega^{\prime} t_{1}, t_{2}$. Consequently, there exists a subsequence that converges almost everywhere to $\nabla u$ in $\Omega^{\prime}{ }_{t_{1}, t_{2}}$. 
Next, by the continuity of $\xi \mapsto \mathcal{A}(x, t, \xi)$, we obtain that $\mathcal{A}\left(x, t, \nabla u_{i}\right)$, $i=1,2, \ldots$, converges to $\mathcal{A}(x, t, \nabla u)$ almost everywhere in $\Omega^{\prime}{ }_{t_{1}, t_{2}}$. Moreover, $\mathcal{A}\left(x, t, \nabla u_{i}\right)$ is uniformly bounded in $L_{\mathrm{loc}}^{p /(p-1)}\left(\Omega_{t_{1}, t_{2}}^{\prime}\right)$. These two facts imply the weak convergence of $\mathcal{A}\left(x, t, \nabla u_{i}\right)$ in $L_{\text {loc }}^{p /(p-1)}\left(\Omega_{t_{1}, t_{2}}^{\prime}\right)$.

Collecting the facts, both terms in the definition of a weak supersolution converge to right limits since $\Omega_{t_{1}, t_{2}}^{\prime} \Subset M_{\tau_{1}, \tau_{2}}$ was arbitrary, proving the assertion.

Actually, the pointwise convergence of the gradients in the proof above implies the strong convergence of the gradients in $L_{\mathrm{loc}}^{p}\left(M_{\tau_{1}, \tau_{2}}\right)$. Indeed, pointwise convergence together with the uniform $L^{p}$-bound implies the strong convergence in $L^{q}$ for any $q$ strictly less than $p$, see, for example, [28]. Nevertheless, due to higher integrability, see Kinnunen-Lewis [26] and also [32], we can repeat the reasoning for $p+\varepsilon$ and, thus, get rid off the restriction $q<p$.

\section{EXISTENCE}

In this section, we prove the existence of a solution to the Cauchy problem. To begin with, we prove the existence of weak solutions for all times when the initial data is in $L^{2}$ and is compactly supported. We next establish the existence for finite compactly supported Radon measures and then extend to the non-compact case where $\nu(M)$ is possibly infinite: We construct a sequence of functions approximating the initial measure and thus, obtain a sequence of approximating solutions. Finally, the compactness result from the previous section completes the proof.

The starting point is to employ the existence theorem for a Dirichlet problem

$$
\left\{\begin{aligned}
\operatorname{div}(\mathcal{A}(x, t, \nabla u)) & =\frac{\partial u}{\partial t} \quad \text { in } \quad \Omega \times(0, T), \\
u(\cdot, 0) & =u_{0} \in L^{2}(\Omega),
\end{aligned}\right.
$$

where

$$
u \in L^{p}\left(0, T ; W_{0}^{1, p}(\Omega)\right) \cap C\left([0, T) ; L^{2}(\Omega)\right)
$$

and $\Omega$ is bounded. In particular, $u$ attains its initial values continuously in $L^{2}$-sense, that is,

$$
\lim _{t \rightarrow 0} \int_{\Omega}\left(u(x, t)-u_{0}(x)\right)^{2} d \mu=0 .
$$

The result is based on a general principle on the monotone operators, see, for example, Lions [30] or Showalter [35], p. 126. See also Alt-Luckhaus [2] and Hüngerbuhler [25].

Theorem 5.3. Let $T>0$ and $\Omega$ be an open bounded subset of $M$. Then there exists a unique weak solution to the Dirichlet problem (5.1).

The existence of the weak solution to the Cauchy problem (2.2) with the $L^{2}$-initial data is more involved.

Theorem 5.4. Suppose that $u_{0} \in L^{2}(M)$ has a compact support. Then there exists a unique solution

$$
u \in L_{l o c}^{p}\left(0, T ; W_{l o c}^{1, p}(M)\right) \cap C\left([0, T) ; L_{l o c}^{2}(M)\right)
$$


to the Cauchy problem. Moreover,

$$
\int_{M} u(x, t) d \mu=\int_{M} u_{0}(x) d \mu
$$

and

$$
\int_{M} \max ( \pm u(x, t), 0) d \mu \leq \int_{M} \max \left( \pm u_{0}(x), 0\right) d \mu
$$

for all $t>0$.

Proof. Suppose that the support of $u_{0}$ belongs to $B\left(x_{0}, R_{0}\right), R_{0}>1$, and let $R \geq 2 R_{0}$. Let $v$ solve (5.1) in $B\left(x_{0}, R\right) \times(0, T), T>0$, with the initial data $v_{0}=u_{0}$. Such a solution exists by Theorem 5.3. The functions $v_{+}=\max (v, 0)$ and $v_{-}=\max (-v, 0)$ are non-negative subsolutions and they attain initial values $\left(u_{0}\right)_{+}$and $\left(u_{0}\right)_{-}$, respectively, by the $L^{2}$-continuity. Moreover, by (3.12), we have

$$
\int_{B\left(x_{0}, R\right)} v_{ \pm}^{2}(x, t) d \mu(x) \leq \int_{B\left(x_{0}, R\right)}\left(u_{0}(x)\right)_{ \pm}^{2} d \mu(x)=: N_{0}^{2}
$$

for all $t>0$. Thus it follows by Hölder's inequality that

$$
\sup _{t \geq 0} f_{B\left(x_{0}, R\right)} v_{ \pm}(x, t) d \mu(x) \leq \frac{N_{0}}{\sqrt{\mu\left(B\left(x_{0}, R\right)\right)}} \leq \frac{N_{0}}{\sqrt{\mu\left(B\left(x_{0}, 1\right)\right)}} .
$$

The second statement in Theorem 3.1 then implies that $v_{ \pm}=0$ almost everywhere near the boundary of $B\left(x_{0}, R\right)$ up to the time $T_{R}$, which has a positive lower bound depending only on $p, \alpha, \beta, C_{p}, C_{\mu}, N_{0}$, and grows linearly with $R^{p}$. Consequently, we can extend the solution $v$ to be zero in the set $\left(M \backslash B\left(x_{0}, R\right)\right) \times\left(0, T_{R}\right)$. Because $R$ is arbitrary, we obtain the existence of the solution in $M \times(0, \infty)$.

The second statement follows easily from the weak formulation. As we have shown, for every fixed $T>0$, the support of $u(\cdot, t), 0<t<T$, belongs to a compact set $K_{t}$. Let $\phi \in C_{0}^{\infty}(M)$ be a time-independent cut-off function such that $\phi=1$ on $K_{t}$. Clearly,

$$
\int_{0}^{t} \int_{M}\left\langle\mathcal{A}\left(x, s, \nabla u_{ \pm}\right), \nabla \phi\right\rangle d \mu d s=0 .
$$

Thus Remark 3.13 implies that

$$
\int_{M} u(x, t) d \mu=\int_{M} u(x, t) \phi(x) d \mu=\int_{M} u_{0}(x) \phi(x) d \mu=\int_{M} u_{0}(x) d \mu
$$

and

$$
\int_{M} u_{ \pm}(x, t) d \mu \leq \int_{M}\left(u_{0}(x)\right)_{ \pm} d \mu
$$

for all $\tau>0$.

Finally, let $u$ be any solution to the Cauchy problem. By the second statement in Theorem 3.1 and Remark 3.5, we may choose for any time $T$ a radius $R$ independent of $u$ such that the support of $u(\cdot, t)$ belongs to $B(0, R)$ for every $0<t<T$. This fact together with the comparison principle implies the uniqueness of the solution. 
In order to use the preliminary result, we need to establish an approximation procedure for finite Radon measures in a sense of weak convergence of measures. This is the content of the following lemma.

Lemma 5.5. Let $\Omega \subset M$ be an open set and let $\nu$ be a finite Radon measure in $\Omega$. Then there exists a sequence $f_{j}, i=1,2, \ldots$, in $C^{\infty}(\Omega)$ such that

$$
\int_{\Omega}\left|f_{j}\right| d \mu \leq|\nu|(\Omega)
$$

and for all $\theta \in C_{0}^{\infty}(\Omega)$ we have

$$
\int_{\Omega} f_{j} \theta d \mu \rightarrow \int_{\Omega} \theta d \nu
$$

as $j \rightarrow \infty$.

Proof. By Vitali's covering argument with a doubling measure, there exists a countable collection of balls $\left\{\widetilde{B}_{i, j}\right\}$ of radius $2^{-j}$ centered in $\Omega$ and of bounded overlap, such that $\Omega \subset \cup_{i=1}^{\infty} \widetilde{B}_{i, j}$ and $\left\{\frac{1}{5} \widetilde{B}_{i, j}\right\}$ is disjoint. Define $\mathcal{F}_{j}=\left\{B_{i, j}\right\}$ to be a collection of those balls in $\left\{\widetilde{B}_{i, j}\right\}$ that are contained in $\Omega$.

We shall employ a partition of unity subordinate to $\mathcal{F}_{j}$, i.e. collection of non-negative smooth functions $\left\{\phi_{i, j}\right\}$, such that $\phi_{i, j}$ is supported in $B_{i, j}$ and $\sum_{i=1}^{\infty} \phi_{i, j}(x)=1$ for all $x \in \cup_{i} B_{i, j}$. We define

$$
f_{i, j}(x)=\left(\int_{B_{i, j}} \phi_{i, j}(y) d \mu(y)\right)^{-1} \int_{B_{i, j}} \phi_{i, j}(y) d \nu(y) \phi_{i, j}(x),
$$

which is a smooth function supported in $B_{i, j}$. We define

$$
f_{j}=\sum_{i=1}^{\infty} f_{i, j} .
$$

Note that for all $i$ and $j$ the inequality

$$
\int_{B_{i, j}}\left|f_{i, j}(x)\right| d \mu(x) \leq \int_{B_{i, j}} \phi_{i, j}(x) d|\nu|(x) .
$$

holds. By this estimate and Lebesgue's monotone convergence theorem, we obtain

$$
\int_{\Omega}\left|f_{j}(x)\right| d \mu(x) \leq \sum_{i=1}^{\infty} \int_{B_{i, j}} \phi_{i, j}(x) d|\nu|(x)=|\nu|(\Omega) .
$$

Next we prove the convergence. To this end, take a function $\theta \in C_{0}^{\infty}(\Omega)$ and $j$ large enough such that $\operatorname{spt} \theta \subset \cup_{i} B_{i, j}$. By the smoothness of $\theta$, there is a constant $C$ depending only on $\theta$, such that

$$
\left|\theta(x)-\theta\left(x_{i, j}\right)\right| \leq C 2^{-j}
$$


for all $x \in B_{i, j}$, where $x_{i, j}$ is the center of $B_{i, j}$. Thus,

$$
\begin{aligned}
& \left|\int_{\Omega} \theta d \nu-\int_{\Omega} f_{j} \theta d \mu\right| \\
& \leq \sum_{i=1}^{\infty}\left|\int_{\Omega} \phi_{i, j} \theta d \nu-\int_{\Omega} \phi_{i, j} \theta\left(x_{i, j}\right) d \nu+\int_{\Omega} \phi_{i, j} \theta\left(x_{i, j}\right) d \nu-\int_{\Omega} f_{i, j} \theta d \mu\right| \\
& \leq 2 C 2^{-j} \sum_{i=1}^{\infty} \int_{\Omega} \phi_{i, j} d|\nu| \leq C 2^{-j}|\nu|(\Omega) .
\end{aligned}
$$

This proves the weak convergence and hence the claim as $j \rightarrow \infty$.

We are now ready to prove existence results for initial measure problems. To begin with, be assume that the initial measure is finite and compactly supported.

Theorem 5.6. Let $\nu$ be a compactly supported Radon measure in $M$. Then there exists a weak solution to the Cauchy problem $(2.2)$ in $M \times(0, \infty)$.

Proof. To prove the theorem, we find a weak solution $u$ in $M \times(0, \infty)$ such that

$$
\int_{M} u(x, t) \varphi(x) d \mu(x) \rightarrow \int_{M} \varphi(x) d \nu(x), \quad \text { as } \quad t \rightarrow 0,
$$

for every $\varphi \in C_{0}^{\infty}(M)$. Let $\Omega$ be an open bounded set in $M$ such that $\nu$ is supported in $\Omega$. In view of Lemma 5.5, there exists a sequence $u_{i}^{0}, i=$ $1,2, \ldots$ of smooth functions in $C^{\infty}(\Omega)$ and

$$
\int_{\Omega}\left|u_{i}^{0}\right| d \mu \leq|\nu|(\Omega)<\infty
$$

Furthermore,

$$
\int_{M} u_{i}^{0} \varphi d \mu \rightarrow \int_{M} \varphi d \nu
$$

as $i \rightarrow \infty$ for every $\varphi \in C_{0}^{\infty}(\Omega)$. Thus, by Theorem 5.4, there exists a weak solution $u_{i} \in L_{l o c}^{p}\left(0, \infty ; W_{l o c}^{1, p}(M)\right) \cap C\left([0, \infty) ; L_{l o c}^{2}(M)\right)$, corresponding to each $u_{i}^{0}$.

Let $t_{0}>0$. According to Theorem 5.4

$$
\sup _{t>0} \int_{M}\left|u_{i}\right| d \mu \leq \int_{\Omega}\left|u_{i}^{0}\right| d \mu \leq|\nu|(\Omega),
$$

and, thus, the first statement in Theorem 3.1 and Remark 3.5 imply that the sequence $u_{i}, i=1,2, \ldots$ is essentially uniformly bounded in $M \times\left(t_{0}, \infty\right)$. Thus, according to Theorem 4.3, there exists a weak solution $u$ in $M \times\left(t_{0}, \infty\right)$ and a subsequence, still denoted by $u_{i}, i=1,2, \ldots$, such that

$$
u_{i} \rightarrow u \text { in } L_{\mathrm{loc}}^{p}\left(M \times\left(t_{0}, \infty\right)\right) .
$$

The result extends to the whole of $M \times(0, \infty)$. Indeed, we can replace $t_{0}$ with $t_{0} / 2$ and repeat the reasoning for the subsequence, which was obtained for $t_{0}$. To continue, we repeat this infinitely many times and finally use the diagonal argument.

Observe that we may lose $L^{2}$-continuity at $t=0$ when taking limits above. Nevertheless, we can show that $u$ takes the right initial values in the sense 
of distributions. Define a linear approximation of the characteristic function as

$$
\chi_{t_{1}, t_{2}}^{h, k}(t)= \begin{cases}0, & t \leq t_{1}-h \\ \left(t+h-t_{1}\right) / h, & t_{1}-h<t<t_{1} \\ 1, & t_{1}<t<t_{2} \\ \left(t_{2}+k-t\right) / k, & t_{2}<t<t_{2}+k \\ 0, & t \geq t_{2}+k,\end{cases}
$$

where $0 \leq t_{1}-h$. Let $\varphi \in C_{0}^{\infty}(M)$ and choose $\varphi(x) \chi_{t_{1}, t_{2}}^{h, k}(t)$ as a test function in the weak formulation. We obtain

$$
\begin{aligned}
\mid f_{t_{2}}^{t_{2}+k} \int_{M} u_{i} \varphi d \mu d t & -f_{t_{1}}^{t_{1}-h} \int_{M} u_{i} \varphi d \mu d t \mid \\
\leq & \left|\int_{t_{1}-h}^{t_{2}+k} \int_{M}\left\langle\mathcal{A}\left(x, t, \nabla u_{i}\right), \chi_{t_{1}, t_{2}}^{h, k} \nabla \varphi\right\rangle d x d t\right| .
\end{aligned}
$$

Next we pass to limits in a particular order. Since $t \mapsto u_{i}(\cdot, t)$ is a continuous function having values in $L^{2}$, it follows that

$$
f_{t_{1}}^{t_{1}-h} \int_{M} u_{i} \varphi d \mu d t \rightarrow \int_{M} u_{i}\left(x, t_{1}\right) \varphi(x) d \mu(x),
$$

as $h \rightarrow 0$. Furthermore, the initial condition implies

$$
\int_{M} u_{i}\left(x, t_{1}\right) \varphi(x) d \mu(x) \rightarrow \int_{M} u_{i}^{0}(x) \varphi(x) d \mu(x),
$$

as $t_{1} \rightarrow 0$. As then $i \rightarrow \infty$, we obtain

$$
\int_{M} u_{i}^{0}(x) \varphi(x) d \mu(x) \rightarrow \int_{M} \varphi(x) d \nu(x),
$$

and

$$
f_{t_{2}}^{t_{2}+k} \int_{M} u_{i} \varphi d \mu d t \rightarrow f_{t_{2}}^{t_{2}+k} \int_{M} u \varphi d \mu d t
$$

due to (5.7) and (5.8). Finally, by passing to zero with $k$, it follows that

$$
f_{t_{2}}^{t_{2}+k} \int_{M} u \varphi d \mu d t \rightarrow \int_{M} u\left(x, t_{2}\right) \varphi(x) d \mu(x),
$$

since the weak solution $u$ belongs to $C\left((0, \infty), L_{\text {loc }}^{2}(M)\right)$. Observe that we only use the continuity on an open interval $(0, T)$.

Consider next the right hand side of (5.9). Let first $h \rightarrow 0$ and $t_{1} \rightarrow 0$ in this order. The growth bounds and (3.4) (see also the remark thereafter) imply

$$
\begin{aligned}
\left|\int_{0}^{t_{2}+k} \int_{M}\left\langle\mathcal{A}\left(x, t, \nabla u_{i}\right), \chi_{0, t_{2}}^{0, k} \nabla \varphi\right\rangle d \mu d t\right| & \leq C \int_{0}^{t_{2}+k} \int_{\operatorname{spt} \varphi}\left|\nabla u_{i}\right|^{p-1} d \mu d t \\
& \leq C\left(t_{2}+k\right)^{1 / \lambda},
\end{aligned}
$$

where $\lambda=d_{\mu}(p-2)+p$ and $C$ depends on $p, \alpha, \beta,|\nu|(\Omega)$ and $\varphi$. Then we pass to limits with $i$ and $k$, merge the estimates, and obtain

$$
\left|\int_{M} u\left(x, t_{2}\right) \varphi(x) d \mu(x)-\int_{M} \varphi(x) d \nu(x)\right| \leq C t_{2}^{1 / \lambda} .
$$


The right hand side tends to zero, as $t_{2} \rightarrow 0$, and we have shown that $u$ takes the right initial values. This completes the proof.

The following theorem extends the existence result for Radon measures with possibly unbounded support. First, we define the seminorm

$$
|\|\nu\||_{\rho}(x):=\sup _{R \geq \rho} R^{-p /(p-2)} \frac{|\nu|(B(x, R))}{\mu(B(x, R))}, \quad x \in M .
$$

We use the abbreviation

$$
|\|\nu\||=\lim _{\rho \rightarrow \infty} \mid\|\nu\|_{\rho}(x) .
$$

The quantity is independent of the choice of $x$ since $M$ is a length space as a geodesic space, and $\mu$ is doubling.

Theorem 5.11. Suppose that $\nu$ is a Radon measure in $M$ and

$$
|\|\nu\||<\infty \text {. }
$$

Then there exists a solution to the Cauchy problem (2.2) up to

$$
T=\frac{1}{C|\|\nu\||^{p-2}},
$$

where the constant $C$ depends only on $p, \alpha, \beta, C_{\mu}$, and $C_{p}$.

Proof. As in the proof Theorem 5.6, we look for a weak solution $u$ in $M \times$ $\left(0,1 /\left(C|\|\nu\||^{p-2}\right)\right.$, such that

$$
\int_{M} u(x, t) \varphi(x) d \mu(x) \rightarrow \int_{M} \varphi(x) d \nu(x) \quad \text { as } \quad t \rightarrow 0,
$$

for every $\varphi \in C_{0}^{\infty}(M)$.

Let $x_{0} \in M$ and let $\chi_{B\left(x_{0}, i\right)}, i=1,2, \ldots$, be a characteristic function of $B\left(x_{0}, i\right)$ and $\nu_{i}=\nu \chi_{B\left(x_{0}, i\right)}$. Let $u_{i}$ be a solution to the corresponding Cauchy problem with the initial trace $\nu_{i}$. We define

$$
N_{\tau}(R)=\sup _{0<t<\tau} f_{B\left(x_{0}, R\right)}\left|u_{i}\right| d \mu \quad \text { and } \quad G_{\tau}(R)=R^{-p /(p-2)} N_{\tau}(R) .
$$

The initial data $\nu_{i}$ is compactly supported, and thus the solution $u_{i}$ exists in $M \times(0, \infty)$, and by assumption (5.12), it follows that $\sup _{R} G(R)<\infty$ for every $\tau>0$.

Let then $\phi \in C_{0}^{\infty}\left(B\left(x_{0}, 2 R\right)\right)$ be a cut-off function such that $\phi=1$ in $B\left(x_{0}, R\right)$ and $|\nabla \phi| \leq C / R$. From the definition of a weak solution (see also Remark 3.13), we obtain

$$
\begin{aligned}
f_{B\left(x_{0}, 2 R\right)} & \left|u_{i}(x, s)\right| \phi(x) d \mu(x) \\
\leq & \frac{\left|\nu_{i}\right|\left(B\left(x_{0}, 2 R\right)\right)}{\mu\left(B\left(x_{0}, 2 R\right)\right)}+\frac{C}{R} \int_{0}^{s} f_{B\left(x_{0}, 2 R\right)}\left|\nabla u_{i}\right|^{p-1} d \mu d t
\end{aligned}
$$

for any $s>0$. We divide this on both sides by $R^{p /(p-2)}$ and take the supremum over the interval $(0, \tau)$. Moreover, since $\left|\nu_{i}\right|\left(B\left(x_{0}, 2 R\right)\right) \leq$ 
$|\nu|\left(B\left(x_{0}, 2 R\right)\right)$, we obtain

$$
\begin{aligned}
G_{\tau}(R) \leq 2^{p /(p-2)}(2 R)^{-p /(p-2)} & \frac{|\nu|\left(B\left(x_{0}, 2 R\right)\right)}{\mu\left(B\left(x_{0}, 2 R\right)\right)} \\
& +C R^{-1-p /(p-2)} \int_{0}^{\tau} f_{B\left(x_{0}, 2 R\right)}\left|\nabla u_{i}\right|^{p-1} d \mu d t .
\end{aligned}
$$

The third statement in Theorem 3.1 together with Remark 3.5 gives

$$
\begin{aligned}
\int_{0}^{\tau} f_{B\left(x_{0}, 2 R\right)}\left|\nabla u_{i}\right|^{p-1} d \mu d t & \leq C\left(\left(R^{d_{\mu}} N_{\tau}(4 R)\right)^{p-2} \tau\right)^{1 / \lambda} N_{\tau}(4 R) \\
& =C R^{1+p /(p-2)} G_{\tau}(4 R)^{1+(p-2) / \lambda} \tau^{1 / \lambda},
\end{aligned}
$$

which holds for any $T>0$ and for every

$$
0<\tau<R^{p}\left(N_{\tau}(4 R)\right)^{2-p}=4^{-p /(p-2)} G_{\tau}(4 R)^{2-p} .
$$

Hence, for such $\tau$, we conclude

$$
G_{\tau}(R) \leq 2^{p /(p-2)}(2 R)^{-p /(p-2)} \frac{|\nu|\left(B\left(x_{0}, 2 R\right)\right)}{\mu\left(B\left(x_{0}, 2 R\right)\right)}+C \tau^{1 / \lambda} G_{\tau}(4 R)^{1+(p-2) / \lambda} .
$$

A careful choice of $\tau$ together with iteration provides a bound for $G_{\tau}(R)$ : The condition (5.14) is certainly satisfied, if we choose $\tau$ according to the equation

$$
\tau=\min \left\{4^{-p /(p-2)},(2 C)^{-\lambda}\right\} G_{\tau}(4 R)^{2-p} .
$$

Such $\tau$ exists since $G_{\tau}(4 R)^{2-p}$ is a continuous decreasing function of $\tau$. Furthermore, by the definition of $|\|\nu\||$, we find for every $\varepsilon>0$ a constant $R_{\varepsilon}$ such that the inequality

$$
|\|\nu\||_{R} \leq|\|\nu\||+\varepsilon
$$

holds for every $R>R_{\varepsilon}$. Collecting the facts, we deduce

$$
G_{\tau}(R) \leq C(|\|\nu\||+\varepsilon)+\frac{1}{2} G_{\tau}(4 R),
$$

for every $R>R_{\varepsilon}$. By iterating (5.16), we end up with

$$
G_{\tau}(R) \leq C(|\|\nu\||+\varepsilon)
$$

for all $R>R_{\varepsilon}$. Consequently, we obtain by (5.15) the lower bound

$$
\tau \geq \frac{1}{C}(|\|\nu\||+\varepsilon)^{2-p}=: T_{\varepsilon} .
$$

Moreover, this implies by the first claim in Theorem 3.1 and Remark 3.5 that

$$
\operatorname{ess~sup}_{B\left(x_{0}, R\right) \times\left(t, T_{\varepsilon}\right)}\left|u_{i}\right| \leq C R^{p /(p-2)}\left(t^{-1 /(p-2)}+|\|\nu\||^{p-1}\right)
$$

for every $0<t<T_{\varepsilon}$ and $R>R_{\varepsilon}$.

We observe that the right hand side of the estimate (5.18) does not depend on $i$, and thus the sequence $u_{i}, i=1,2, \ldots$ is uniformly bounded in $B\left(x_{0}, R\right) \times\left(t, T_{\varepsilon}\right)$. Consequently, there exists a subsequence still denoted by $u_{i}, i=1,2, \ldots$ and a weak solution $u$ such that $u_{i} \rightarrow u$ in $L_{\mathrm{loc}}^{p}\left(B\left(x_{0}, R\right) \times\left(t, T_{\varepsilon}\right)\right)$ by Theorem 4.3. We can repeat the argument with any finite $R>R_{\varepsilon}$ and also for any $t=2^{-k} T_{\varepsilon}, k \in \mathbb{N}$. Thus, the function $u$ 
is a weak solution in $M \times\left(x_{0}, T\right)$ by the diagonal argument, $\mathrm{cf}$. the proof of Theorem 5.6. By letting $\varepsilon$ to zero, we obtain the first part of the claim.

It remains to show that $u$ takes the right initial values. First, choose $\varphi \in C_{0}^{\infty}(M)$. Similarly as in the derivation of estimate (5.10), the third claim in Theorem 3.1, Remark 3.5, and (5.17) imply

$$
\left|f_{t_{2}}^{t_{2}+k} \int_{M} u_{i}(x, t) \varphi(x) d \mu(x) d t-\int_{M} \varphi(x) d \nu_{i}(x)\right| \leq C t_{2}^{1 / \lambda}
$$

where spt $\varphi \subset B\left(x_{0}, R\right)$ and $C$ depends on $p, \alpha, \beta, R,|\|\mu\||, \varepsilon$, and $\varphi$. Since $\nu_{i}=\nu$ in $\operatorname{spt} \varphi$ for $i$ large enough, it follows that

$$
\left|\int_{M} u\left(x, t_{2}\right) \varphi(x) d \mu(x)-\int_{M} \varphi(x) d \nu(x)\right| \leq C t_{2}^{1 / \lambda},
$$

as $i \rightarrow \infty$ and $k \rightarrow 0$. Letting $t_{2} \rightarrow 0$ completes the proof.

\section{Optimality of the eXistence Result}

In this section, we show in the Euclidean setting that if the weak solution exists, then the initial trace satisfies the assumptions of the previous section. In particular, the existence fails with more general measures and thus our results are sharp. The proof is based on the following global weak Harnack inequality, see [18], [29], as well as [14] and [19].

Theorem 6.1. Let $u$ be a non-negative weak supersolution in $\mathbb{R}^{n} \times(0, T)$. Then there exists a constant $C=C(n, p, \alpha, \beta)$ such that for every $x_{0} \in \mathbb{R}^{n}$, $R>0$ and almost every $0<t<T / 4$, we have

$$
f_{B\left(x_{0}, R\right)} u(x, t) d x \leq\left(\frac{C R^{p}}{T}\right)^{1 /(p-2)}+C\left(\frac{T}{R^{p}}\right)^{n / p} \underset{Q}{\operatorname{essinf}} u^{\lambda / p},
$$

where $\lambda=n(p-2)+p$ and $Q=B\left(x_{0}, 2 R\right) \times(T / 2, T)$.

Observe that if $u$ is a solution, then the result holds for every $0<t<T / 4$.

Theorem 6.2. Suppose that $u$ is a non-negative weak solution in $\mathbb{R}^{n} \times(0, T)$. Then there is a unique non-negative locally finite Radon measure $\nu$ such that

$$
\lim _{t \rightarrow 0} \int_{\mathbb{R}^{n}} u(x, t) \varphi(x) d x \rightarrow \int_{\mathbb{R}^{n}} \varphi(x) d \nu(x)
$$

for every $\varphi \in C_{0}^{\infty}\left(\mathbb{R}^{n}\right)$. Moreover, there exists a constant $C=C(n, p, \alpha, \beta)$ such that

$$
|\|\nu\|| \leq C T^{-1 /(p-2)}
$$

Proof. To begin with, choose $B(0, R)$ and a sequence $\tau_{i}, i=1,2, \ldots$ tending to zero. By the global Harnack estimate, Theorem 6.1, it follows that $u\left(x, \tau_{i}\right) d x, i=1,2, \ldots$ is a bounded sequence of positive Radon measures in $B(0, R)$. Thus, the weak compactness for measures implies that there exists a positive Radon measure $\nu$ such that (6.3) holds for a subsequence. The diagonal argument with $R=1,2, \ldots$ extends the result to the whole of $\mathbb{R}^{n}$.

It remains to show the uniqueness of $\nu$. Theorem 6.1 implies for every $R \geq 1$ and $0<t<T / 4$ that

$$
R^{-p /(p-2)} f_{B(0, R)} u(x, t) d x \leq\left(\frac{C}{T}\right)^{1 /(p-2)}+\frac{C T^{n / p}}{R^{\lambda /(p-2)}} \underset{Q}{\operatorname{essinf}} u^{\lambda / p},
$$


where $Q=B(0,2 R) \times(T / 2, T)$ and $\lambda=n(p-2)+p$. By taking the essential supremum in (6.4) with respect to $t$ over the interval $(0, T / 4)$ and adopting the notation from the proof of Theorem 5.11, we conclude that $G_{T / 4}(R)$ is bounded. Furthermore, similarly as in the proof of Theorem 5.11, we obtain for any $\varphi \in C_{0}^{\infty}\left(\mathbb{R}^{n}\right)$ and for all $0<t_{1}, t_{2}<T / 4$ that

$$
\left|\int_{\mathbb{R}^{n}} u\left(x, t_{2}\right) \varphi(x) d x-\int_{\mathbb{R}^{n}} u\left(x, t_{1}\right) \varphi(x) d x\right| \leq C\left(t_{2}-t_{1}\right)^{1 / \lambda} .
$$

Observe that $C$ does not depend on $t_{1}$ or $t_{2}$. By replacing $t_{1}$ by $\tau_{i}$ and passing to a limit with $i$, we deduce that the initial trace is unique. The estimate for $|\|\nu\||$ follows from (6.4) by letting $t \rightarrow 0$ and $R \rightarrow \infty$.

\section{REFERENCES}

[1] E. Acerbi and G. Mingione. Gradient estimates for a class of parabolic systems. Duke Math. J., 136(2):285-320, 2007.

[2] H. W. Alt and S. Luckhaus. Quasilinear elliptic-parabolic differential equations. Math. Z., 183(3):311-341, 1983.

[3] F. Andreu, J. M. Mazón, S. Segura de León, and J. Toledo. Existence and uniqueness for a degenerate parabolic equation with $L^{1}$-data. Trans. Amer. Math. Soc., 351(1):285-306, 1999.

[4] D. G. Aronson. Widder's inversion theorem and the initial distribution problems. SIAM J. Math. Anal., 12(4):639-651, 1981.

[5] D. G. Aronson and L. A. Caffarelli. The initial trace of a solution of the porous medium equation. Trans. Amer. Math. Soc., 280(1):351-366, 1983.

[6] D. Bakry, T. Coulhon, M. Ledoux, L. Saloff-Coste, Sobolev inequalities in disguise. Indiana Univ. Math. J. 44, 1033-1074, 1995.

[7] G.I. Barenblatt, On selfsimilar motions of compressible fluids in porous medium (in Russian). Prikl Mat. Mekh. 16, 679-698, 1952.

[8] P. Bénilan, M. G. Crandall, and M. Pierre. Solutions of the porous medium equation in $\mathbf{R}^{N}$ under optimal conditions on initial values. Indiana Univ. Math. J., 33(1):5187, 1984 .

[9] D. Blanchard and F. Murat. Renormalised solutions of nonlinear parabolic problems with $L^{1}$ data: existence and uniqueness. Proc. Roy. Soc. Edinburgh Sect. A, 127(6):1137-1152, 1997.

[10] L. Boccardo, A. Dall'Aglio, T. Gallouët, and L. Orsina. Nonlinear parabolic equations with measure data. J. Funct. Anal., 147(1):237-258, 1997.

[11] L. Boccardo and T. Gallouët. Nonlinear elliptic equations with right-hand side measures. Comm. Partial Differential Equations, 17(3-4):641-655, 1992.

[12] M. Bonforte and G. Grillo. Asymptotics of the porous media equation via Sobolev inequalities. J. Funct. Anal., 225(1):33-62, 2005.

[13] I. Chavel, Riemannian geometry - a modern introduction, Cambridge Tracts in Mathematics, vol. 108, Cambridge University Press, Cambridge, 1993.

[14] H. J. Choe and J. H. Lee. Cauchy problem for nonlinear parabolic equations. Hokkaido Math. J., 27(1):51-75, 1998.

[15] B. E. J. Dahlberg and C. E. Kenig. Nonnegative solutions of the porous medium equation. Comm. Partial Differential Equations, 9(5):409-437, 1984.

[16] S. A. J. Dekkers. Finite propagation speed for solutions of the parabolic $p$-Laplace equation on manifolds. Comm. Anal. Geom., 13(4):741-768, 2005.

[17] E. DiBenedetto. Degenerate parabolic equations. Universitext. Springer-Verlag, New York, 1993.

[18] E. DiBenedetto, U. Gianazza, and V. Vespri. Harnack Estimates for Quasi-Linear Degenerate Parabolic Differential Equation. Acta Math., 200(2):181-209, 2008.

[19] E. DiBenedetto, U. Gianazza, and V. Vespri. Potential-like lower bounds for nonnegative solutions to certain quasi-linear degenerate parabolic differential equations, 
and applications to alternative forms of the harnack inequality. Duke Math. J., 143(1):1-15, 2008.

[20] E. DiBenedetto and M. A. Herrero. On the Cauchy problem and initial traces for a degenerate parabolic equation. Trans. Amer. Math. Soc., 314(1):187-224, 1989.

[21] M. P. do Carmo. Riemannian geometry. Mathematics: Theory \& Applications. Birkhäuser Boston Inc., Boston, 1992.

[22] M. Giaquinta. Introduction to regularity theory for nonlinear elliptic systems. Lectures in Mathematics ETH Zürich. Birkhäuser Verlag, Basel, 1993.

[23] A. Grigor'yan, The heat equation on non-compact Riemannian manifolds. Matem. Sbornik 182, 55-87, 1991. Engl. Transl. Math. USSR Sb. 72, 47-77, 1992

[24] E. Hebey, Nonlinear analysis on manifolds: Sobolev spaces and inequalities, Courant Lecture Notes in Mathematics, vol. 5, New York University Courant Institute of Mathematical Sciences, New York, 1999.

[25] N. Hungerbühler. Quasi-linear parabolic systems in divergence form with weak monotonicity. Duke Math. J., 107(3):497-520, 2001.

[26] J. Kinnunen and J. L. Lewis. Higher integrability for parabolic systems of $p$-Laplacian type. Duke Math. J., 102(2):253-271, 2000.

[27] J. Kinnunen and N. Shanmugalingam. Regularity of quasi-minimizers on metric spaces. Manuscripta Math., 105(3):401-423, 2001.

[28] R. Korte, T. Kuusi, and M. Parviainen. A connection between a general class of superparabolic functions and supersolutions. Submitted, 2008.

[29] T. Kuusi. Harnack estimates for weak supersolutions to nonlinear degenerate parabolic equations. Ann. Scuola Norm. Sup. Pisa Cl. Sci. (5), 7(4):1-44, 2008.

[30] J-L. Lions. Quelques méthodes de résolution des problèmes aux limites non linéaires. Dunod, 1969.

[31] J.J. Manfredi and V. Vespri. Large time behavior of solutions to a class of doubly nonlinear parabolic equations. Electron. J. Differential Equations, 2-17, 1994.

[32] M. Parviainen. Global gradient estimates for degenerate parabolic equations in nonsmooth domains. To appear in Ann. Mat. Pura Appl., doi:10.1007/s10231-008-0079-0.

[33] J.-M. Rakotoson. A compactness lemma for quasilinear problems: application to parabolic equations. J. Funct. Anal., 106(2):358-374, 1992.

[34] L. Saloff-Coste, Aspects of Sobolev-type inequalities. London Mathematical Society Lecture Note Series 289, Cambridge University Press, 2002.

[35] R. E. Showalter. Monotone operators in Banach space and nonlinear partial differential equations. volume 49 of Mathematical Surveys and Monographs, American Mathematical Society, Providence, RI, 1997.

[36] J. Simon. Compact sets in the space $L^{p}(0, T ; B)$. Ann. Mat. Pura Appl. (4), 146:6596, 1987.

[37] J. L. Vázquez. The porous medium equation. Oxford Mathematical Monographs, The Clarendon Press, Oxford University Press, Oxford, 2007

[38] D. V. Widder. Positive temperatures on an infinite rod. Trans. Amer. Math. Soc., 55:85-95, 1944. 